\title{
EL PARTITO DEMOCRATICO: DEL PARTIDO DE LOS REFORMISTAS AL PARTIDO FALLIDO. UN PRIMER BALANCE (2007-2019)
}

\section{Partito Democratico: From reformist's party to failed party. A first Assessment (2007-2019)}

\author{
LUCIANO M. FASANO \\ Università degli studi di Milano \\ luciano.fasano@unimi.it
}

Cómo citar/Citation

Fasano, L. M. (2020).

El Partito Democratico: del partido de los reformistas al partido fallido. Un primer balance (2007-2019).

Revista de Estudios Políticos, 189, 127-166. doi: https://doi.org/10.18042/cepc/rep.189.05

\section{Resumen}

La historia del Partito Democratico (PD) ofrece un ejemplo importante de las transformaciones que han llamado la atención sobre el sistema político y de partidos italiano en el curso de la última década. Nacido de la fusión de dos de los principales partidos de la coalición de centro-izquierda, DS y La Margherita, que durante veinte años han sido protagonistas junto al centro-derecha de Berlusconi de la evolución en sentido bipolar de la política italiana, una docena de años después de su acto de nacimiento, el PD es un partido cuyo proyecto político debe considerarse todavía indeterminado e incompleto. El presente artículo delinea un primer balance de esta experiencia política a través de la reconstrucción de las diversas fases políticas de la vida política del PD, desde su nacimiento en 2007 hasta 2019, tratando de indicar las principales causas que han condicionado sus vicisitudes, desde sus inicios en las elecciones de 2008 hasta la derrota electoral de 2018 y la limitada recuperación registrada en las elecciones europeas y regionales de 2019.

1 Traducción del texto por Jorge del Palacio Martín. 


\section{Palabras clave}

Sistema político italiano; sistema de partidos; partidos políticos; Partito Democratico; elecciones; primarias.

\section{Abstract}

The story of the Partito Democratico provides an important glimpse of the transformations that have affected the Italian political and party system over the past decade. Born by merger of the two main parties of the center-left coalition, DS and Margherita, which for twenty years have been protagonists together with Berlusconi's center-right in the bipolar evolution of Italian politics, a dozen years after its birth act, the PD is a party whose political project has yet to be considered undetermined and uncomplete. In this article a first balance of this experience is outlined, through the reconstruction of the different political phases lived by the PD, from his birth act (2007) to 2019, trying to indicate the main causes that have so far influenced its alternating events, from the debut to 2018 National elections until the electoral defeat of 2018 and the limited recovery of consensus that occurred in the 2019 European and Regional elections.

\section{Keywords}

Italian political system; party system; political party; Partito Democratico; political elections; primaries. 


\section{SUMARIO}

I. INTRODUCCIÓN: 1. Nota metodológica sobre las fuentes de datos. II. LOS ORÍGENES DEL PARTITO DEMOCRATICO: 1. De la experiencia de L'Ulivo a la unidad de las fuerzas reformistas. 2. El difícil compromiso entre almas políticas diferentes. 3. El nacimiento del PD: de las primarias a la fusión fría. III. LAS CUATRO FASES DE LA HISTORIA DEL PARTITO DEMOCRATICO: 1. El partido amalgama de Walter Veltroni. 2. El partido old style de Bersani. 3. El partido pragmático de Renzi. 4. El partido ómnibus de Zingaretti. IV. EVOLUCIÓN DEL CONSENSO ELECTORAL DEL PD. V. UN PARTIDO DIVIDIDO: TRES ALMAS DISTINTAS Y UN CONFLICTO IRRESOLUBLE. VI. AFILIADOS Y SELECTORES. VII. CONCLUSIONES: QUÉ NO HA FUNCIONADO HASTA AHORA EN EL PD. BIBLIOGRAFÍA.

\section{INTRODUCCIÓN}

Entre la primavera y el otońo de 2007, cuando en Italia la idea de una nueva fuerza unitaria de centro-izquierda - que iba a llamarse Partito Democratico (PD) — estaba tomando forma, las expectativas de los ciudadanos y de la opinión pública, no menos que la atención de los observadores y especialistas, eran muy elevadas. Tras el hundimiento de la llamada Primera república, las coaliciones de centro-derecha y centro-izquierda que se alternaron en el poder se caracterizaron por un elevado nivel de fragmentación (Fasano y Pasini, 2014), factor que impedía a los Gobiernos mantener una línea programática - con la que habían conseguido, de otra parte, el consenso de los electores- de manera coherente y sostenida. Los continuados chantajes de las fuerzas políticas menores de ambos bandos, en perjuicio de las fuerzas mayoritarias de las respectivas coaliciones, comprometían cualquier acción reformista del Gobierno de turno para el futuro económico y social del país (Almagisti et al., 2014). En este escenario, el surgimiento de un partido unitario de los reformistas de centro-izquierda, que a su vez favorecería el nacimiento de un partido unitario del centro-derecha moderado, el Popolo della Libertà — nacido de la fusión de Forza Italia y Alleanza Nazionale- fue considerado un factor importante de simplificación del intrincado cuadro político italiano, que andando el tiempo debería favorecer la alternancia de Gobiernos más cohesionados y estables. 
El nacimiento de esta nueva formación política parecía llegar, sin embargo, con retraso. Un hecho que ha marcado de manera determinante una historia de poco más de una década. El final de la llamada Primera república, que implosionó como consecuencia de los escándalos del caso judicial Tangentopoli, vino en 1993. En adelante los principales protagonistas del centro-izquierda italiano, PDS y DS — como herederos de la tradición comunista- y el PPI y Democratici e Libertà-La Marguerita — como herederos de la tradición democristiana-, se encontraron de manera estable en el mismo bando político, compartiendo candidaturas a la premiership y responsabilidad de gobierno. Sin embargo, no fueron capaces de dar una salida eficaz y consecuente a la experiencia de L'Ulivo con la que Romano Prodi venció en las elecciones de 1996. El proceso de construcción de un sujeto político unitario de centro-izquierda, de carácter reformista y con vocación mayoritaria y de gobierno, podría haber contribuido a canalizar la larga e incompleta transición italiana, favoreciendo, sobre todo, una aproximación eficaz a una democracia competitiva y de alternancia. Sin embargo, las cosas se han sucedido de modo distinto y el balance de la trayectoria del PD no puede considerarse más que contradictorio, con luces y sombras, y lejos de las expectativas generadas en su fase constituyente.

El objetivo del presente artículo es reconstruir la historia del PD, esbozando un primer balance de sus doce años de vida. Tal reconstrucción tratará, sobre todo, de poner de manifiesto las características políticas y organizativas del partido en correspondencia con los estilos de liderazgo reconocibles en los cuatro secretarios elegidos por el mecanismo de las primarias. El análisis de estos aspectos se integrará en el examen de la evolución del consenso electoral y del estudio de las características sociodemográficas, como de la orientación política, de dirigentes, delegados nacionales, militantes y selectores (a saber, los electores que han participado en las primarias) del partido. Esta aproximación permitirá delinear las diferentes facetas de un partido que, dividido al menos en tres almas políticas distintas e irreducibles, permanece incompleto al no haber conseguido aún consolidarse en torno a una cultura política y un modelo organizativo compartido, capaz de permitir una institucionalización estable como sujeto político unitario. Precisamente, esta ausencia de institucionalización está en el origen de las múltiples escisiones que en el curso del tiempo han contribuido a debilitar el partido frente a sus grupos dirigentes, en relación con el cuadro de la militancia activa o su electorado.

En este sentido, el PD, como veremos, puede definirse como un partido fallido: un partido incapaz de definir una síntesis o modus vivendi pacífico entre las distintas culturas políticas que lo componen y, como consecuencia, incapaz de dirigir a su electorado un mensaje reconocible capaz de representar el punto de referencia común que la izquierda italiana ha necesitado. Como tampoco, del mismo modo, ha conseguido convertirse en un elemento de 
estabilidad para un sistema político que, saliendo de una fase de transición que dura veinticinco años sin alumbrar una solución de carácter institucional, se encuentra experimentando una fragmentación del sistema de partidos y una polarización ideológica más acentuada que en el pasado.

\section{NOTA METODOLÓGICA SOBRE LAS FUENTES DE DATOS}

Los datos utilizados en el artículo, aparte de los oficiales derivados de los resultados electorales ${ }^{2}$, provienen de las siguientes fuentes:

- Militantes del PD. Encuesta realizada en marzo y abril de 2013 sobre una muestra de 13650 individuos por Candidate and Leader Selection (C\&LS), standing group de la Società Italiana di Scienza Politica (SISP) sobre las elecciones primarias y mecanismos de selección de candidatos en Italia.

- Electores de las primarias del PD (selectores). Entrevistas efectuadas sobre una muestra de 4000 electores en cada una de las consultas primarias, archivo de sondeos del Candidate and Leader Selection (C\&LS).

- Delegados de la Asamblea Nacional del PD. Entrevistas a los delegados efectuadas en cada una de las tomas de posesión de las asambleas nacionales elegidas en relaciones a las primarias, bajo la dirección de Luciano M. Fasano y Paolo Natale, Archivo de Encuestas del Departamento de Ciencias Políticas y Sociales de la Università degli Studi di Milano.

—Dirección nacional del PD. Datos oficiales facilitados por el Partito Democratico.

\section{LOS ORÍGENES DEL PARTITO DEMOCRATICO}

\section{DE LA EXPERIENCIA DE L'ULIVO A LA UNIDAD DE LAS FUERZAS REFORMISTAS}

El proceso constituyente del Partito Democratico (PD) se inicia como consecuencia de los congresos celebrados por Democratici di Sinistra y Democrazia e Libertà-La Margherita entre el 19 y el 21 de abril de 2007, en Florencia

2 Fuente: ELIGENDO, base de datos sobre las elecciones en Italia del Ministerio del Interior. 
y Roma respectivamente. En dichos congresos ambas organizaciones deciden disolverse para dar vida a un nuevo sujeto político. En realidad, sin embargo, se trata de una decisión que viene de lejos y que remite, en última instancia, a la experiencia de L'Ulivo. La coalición liderada por Romano Prodi con la que el centro-izquierda italiano concurrió a las elecciones de 1996. Podemos afirmar, de hecho, que la experiencia de L'Ulivo representaba in nuce el embrión del PD. Dejados atrás los años de la Guerra Fría y la caída el muro de Berlín, después del proceso judicial Tangentopoli, que había destruido los principales partidos de la llamada Primera república —empezando por los partidos de Gobierno (DC, PSI, PSDI, PLI, PRI)—, dejando indemne en parte al PCI, tomó cuerpo la idea de llegar a un acuerdo entre las dos tradiciones políticas y culturales que habían tenido una mayor influencia en la experiencia republicana: la católico democrática y la socialcomunista. Una suerte de reedición del llamado compromiso histórico, que a medidos de los ańos setenta había llevado, con el impulso de Aldo Moro y Enrico Berlinguer, al acercamiento entre la DC y el PCI. Con la consecuencia de un primer, si bien breve, ingreso de los comunistas en la esfera de gobierno.

La posibilidad de un nuevo partido de este tipo también había sido planteada en el pasado por un intelectual de referencia de la izquierda italiana, el economista Michele Salvati. En 2003, en el periódico Il Foglio, Salvati publicó un texto titulado "Appello per il Partito democratico" en el que proponía abiertamente la necesidad del nacimiento de un nuevo sujeto político. En palabras de Salvati, el partido debía ser «la reunión de todos las corrientes reformistas y moderadas de la historia italiana de las que tanto se ha hablado a propósito de L'Ulivo» ${ }^{3}$. Sin embargo, la referencia a la experiencia original de L'Ulivo, en tanto que nueva casa de los católicos democráticos y los socialcomunistas, no agota las razones que llevaron a la experiencia del nacimiento del PD en 2007 como nuevo partido del centro-izquierda italiano.

Las elecciones de 2006 fueron testigo de la victoria, diez años después, de una coalición liderada por Romano Prodi, L'Unione. Tal victoria, empero, se concretó en poco más de 24000 votos frente a la coalición de centro-derecha liderada por Silvio Berlusconi. Esta nueva experiencia de gobierno del centro-izquierda italiano se puso en marcha con muchísimas dificultades. Ya fuera por la exigua mayoría de la que gozaba en el Senado, como por los continuos conflictos que surgieron dentro de la propia coalición. Siempre en torno a cuestiones fundamentales para la acción del Ejecutivo, tales como la política economía o la política exterior. El hecho, asimismo, de que el símbolo de L'Ulivo hubiese sumado más votos en la Cámara de los

3 Cfr. Il Foglio, 10-03-2003. 
Diputados $(31,2 \%)$ que la suma de los votos obtenidos por DS y La Margherita en el Senado, planteaba con fuerza, nuevamente, la posibilidad de dar vida a un gran partido unitario que acogiese a los reformistas de centro-izquierda ${ }^{4}$. De modo que este proceso se inició después de las elecciones de 2006 con la creación del grupo L'Ulivo - que comprendía a los representantes de DS y de La Margherita en las dos ramas del Parlamento italiano-y la convocatoria de los congresos nacionales de los dos partidos, arriba citados, que pusieron en marcha los mecanismos que llevarían al proceso constituyente del nuevo partido. El primer paso después de las citas congresuales era la nominación del comité que debía promover la elección del secretario del nuevo partido y la Asamblea Nacional.

Finalmente, otro elemento catalizador del nacimiento del PD fue el resultado de las elecciones administrativas de 2007, celebradas poco después de los congresos de Florencia y Roma, en las que la derrota de DS y La Margherita, junto a los candidatos a alcalde de L'Unione, sobre todo en las provincias del norte de Italia, fue clara ${ }^{5}$. La reacción de los grupos dirigentes de los dos partidos no se hizo esperar y el proceso de construcción del PD sufrió una aceleración suplementaria. El esfuerzo se concretó en la candidatura de Walter Veltroni, exalcalde de Roma y secretario de Democratici di Sinistra entre 1998 y 2001, a liderar el nuevo partido, convirtiéndose en el primer front runner del PD gracias al apoyo de la mayoría de los dos partidos fundadores.

\section{EL DIFÍCIL COMPROMISO ENTRE ALMAS POLÍTICAS DIFERENTES}

Desde un punto de vista cultural, antes incluso que político, el horizonte de un partido único de los reformistas de carácter democrático, capaz de superar las divisiones ideológicas novecentistas para presentar su candidatura al gobierno del país de forma creíble, dirigiéndose abiertamente a las expectativas y necesidades de todos los electores, parecía responder a una necesidad histórica. Una exigencia que el politólogo inglés Henry Drucker, atento observador del laborismo inglés, ya había puesto sobre la mesa hace alguna década.

4 La decisión de presentar una lista unitaria a la Cámara de los Diputados en las elecciones de 2006 fue tomada tras la experiencia positiva de la lista Uniti nell'Ulivo presentada conjuntamente por Ds y La Margherita a las elecciones al Parlamento Europeo de 2004, en la que obtuvo er $31,9 \%$ de los votos.

5 La coalición de centro-izquierda, bajo el símbolo de L'Unione, perdió ciudades importantes como Verona, Monza o Alessandria, además de las provincias de Vercelli y Gorizia. 
A finales de los años setenta, Drucker había identificado la coexistencia de tres almas político-culturales distintas en el seno del Labour Party ${ }^{6}$. Una combinación que también podía detectarse con claridad en el caso de los partidos de la izquierda italiana. En primer lugar, un alma de carácter ético, de inspiración marxista, fundada en la lucha contra las desigualdades, el igualitarismo, la aspiración a redimir a los sectores más débiles de la sociedad, la defensa de los pobres y los desheredados y, finalmente, sobre una lógica de afirmación universal de los derechos humanos. En segundo lugar, un alma socialdemócrata o laborista, mayormente ligada al propio electorado de referencia, a saber, en su evolución histórica, desde el proletariado a la clase obrera y trabajadora general, caracterizada por un tipo de acción orientada a garantizar mayores oportunidades sociales y económicas a través de la conquista de un Estado de bienestar progresivamente más extenso. En tercer lugar, un alma reformista en sentido liberal-democrático, orientada pragmáticamente al objetivo de gobernar el país a través de un consenso electoral amplio, sirviéndose de instrumentos y modos de acción que, entendiendo el crecimiento económico como la verdadera prioridad estratégica, combina Estado y mercado, sin desdeñar, por tanto, soluciones de tipo liberal o libertario.

La prevalencia de un alma sobre las demás — siempre según el politólogo inglés - ha terminado por condicionar los objetivos, la acción política, el posicionamiento estratégico y las posibilidades de gobernar de los partidos. Cuando han prevalecido la primera y la segunda sobre la tercera, se ha dado una mayor posibilidad de que el partido permanezca en la oposición, persiguiendo como objetivo prioritario condicionar la iniciativa del Gobierno de turno. Viceversa, cuando la tercera se afirma sobre las demás lleva a la fuerza de izquierda a ponerse como objetivo gobernar el país, entrando en competición con las fuerzas moderadas de derecha o centro-derecha. En el primer caso, el partido se condena a representar exclusivamente un área no mayoritaria, electoralmente hablando, de la sociedad. Un área que se corresponde, grosso modo, con el mundo del trabajo vinculado a la gran industria, de cuyos intereses se hace cargo. En el segundo, la izquierda se refiere como electorado a la sociedad, de manera integral, con el objetivo puesto en la transformación del país, pero a través de propuestas abiertas a la mayoría de la sociedad.

Este análisis de Drucker se adaptaba de manera prácticamente perfecta a la situación de la izquierda italiana de la llamada Segunda república. Un análisis, por lo demás, que encontraba plena correspondencia con la valoración que la izquierda merecía a Salvati en el artículo de 2003 citado anteriormente: «A prescindir de las grandes narraciones, los diseños de reorganización radical de la

6 Cfr. Drucker (1979). 
economía y de la sociedad, la izquierda debe volver a la gran tradición moderna, la tradición liberal, a la idea del empoderamiento del mayor número posible de ciudadanos $[\ldots]$ para construir una nueva identidad de izquierda liberal» ${ }^{7}$.

\section{EL NACIMIENTO DEL PD: DE LAS PRIMARIAS A LA FUSIÓN FRÍA}

El 14 de octubre de 2007, aún con el frágil y dividido Gobierno de Prodi en el cargo, Walter Veltroni fue elegido primer secretario del PD. Su elección se produjo a través de una consulta abierta a todos los electores dispuestos a suscribir la "Carta dei valori» del neonato partido. Una consulta que los promotores definieron, desde el inicio - impropiamente- como "primarias». Sin embargo, cuando menos desde el punto de vista político, la denominación no podía considerarse del todo errónea. Resulta cierto que las primarias, en cuanto tales, son un mecanismo para la elección de candidatos a cargos institucionales. Pero es cierto, del mismo modo, que el Estatuto del PD, aprobado al poco tiempo, había previsto la posibilidad de primarias de coalición para la elección del candidato a la presidencia del Consejo de Ministros y reconocía el principio de identificación entre party leadership y la candidatura a la premiership, convirtiendo, por tanto, las consultas para la elección del secretario del partido en instrumento para la elección del candidato al Palazzo Chigi del centro-izquierda.

El PD nacía, de este modo, impulsado por la fuerza de los acontecimientos externos y con el objetivo de afrontar una situación de emergencia, como el mismo Veltroni dejaría por escrito en un artículo publicado en L'Unità años después de poner en marcha el nuevo partido ${ }^{8}$. La llamada fusión fría -a saber, el nacimiento del PD a partir de una reorganización in vitro diseñada por los aparatos de poder postdemocristianos y postcomunistas-, era la característica más distintiva y, al mismo tiempo, el límite más evidente del nuevo partido. En este sentido, las llamadas primarias, abiertas a los electores del centro-izquierda, fueron instituidas con el objetivo de impedir que los grupos dirigentes de los dos partidos fundadores pudiesen representar una legado demasiado determinante en el proceso de construcción del nuevo partido. Sin embargo, el recurso se mostraría insuficiente para conjurar este peligro. La misma candidatura de Veltroni debía su fuerza al apoyo de los aparatos de los partidos fundantes, más que, por ejemplo, al sindicato mayoritario de los trabajadores italianos, la CGIL. Estos elementos configuraron una herencia difícil de manejar en la construcción del nuevo sujeto político,

7 Cfr. Il Foglio, 10-03-2003.

8 Cfr. l'Unità, 14-10-2016. 
condicionando no solo las dinámicas de selección de candidatos y grupos dirigentes, sino también comprometiendo la elaboración de una cultura política propia.

\section{LAS CUATRO FASES DE LA HISTORIA DEL PARTITO DEMOCRATICO}

A partir de su fundación, la historia del PD se desarrollará sustancialmente en cuatro fases. Cada una de las cuales encuentra su correspondencia y carácter distintivo en la figura del secretario elegido a través del mecanismo de las primarias. Podemos, por tanto, identificar: una primera fase, la del partido amalgama, vinculada al liderazgo de Walter Veltroni; una segunda fase, la del partido socialdemócrata de viejo estilo ligado al liderazgo de Pierluigi Bersani; una tercera fase, la del partido pragmático ligado al liderazgo de Matteo Renzi, y una cuarta fase, la actual del partido ómnibus, vinculado al liderazgo de Nicola Zingaretti ${ }^{9}$ A continuación, procederemos a su análisis, en rápida sucesión, para una mejor comprensión de la evolución del partido.

\section{EL PARTIDO AMALGAMA DE WALTER VELTRONI}

El bautismo del PD, con la elección de Veltroni como secretario con el $75 \%$ de los votos (contra el 13\% de Rosy Bindi y el 12\% de Enrico Letta), fue celebrado con la participación de más de tres millones y medio de electores en las primarias del 14 de octubre de 2007. El mal estado de salud del Gobierno Prodi y el emergente liderazgo de Veltroni, destinado a ser el candidato a premier del PD, aceleraron el camino hacia nuevas elecciones ${ }^{10}$. La inminente vuelta a las urnas de los italianos obligaba al nuevo partido a darse una estructura organizativa de manera rápida y eficaz. La fase constituyente, sin embargo, estuvo marcada por el problema de la unificación interna, tanto de grupos dirigentes como de programas, bajo el liderazgo de Veltroni, un líder que por

9 Para una reconstrucción amplia del decenio de vida del PD con referencia a las tres imágenes del partido, véanse Natale y Fasano (2017). En referencia al partido omnibus de Zingaretti se recomienda Fasano y Natale (2019). Entre las contribucions más relevantes sobre el expediente del PD, véanse Salvati (2003), Macaluso (2007), Funiciello (2012), Fassino (2018), Ariemma (2014), Tedeschi (2018) y Floridia (2019).

10 Tras la crisis de la mayoría de gobierno que se produce en 2008 con la dimisión de Clemente Mastella (Udeur) del cargo de ministro de Justicia, el Gobierno Prodi se vio obligado a dimitir. 
historia política y características personales se distinguía como un político fuertemente orientado hacia la búsqueda de unidad y consenso.

Entre los primeros debates, el referido al modelo de partido fue particularmente intenso, respecto al cual se delinearon dos visones claramente opuestas. De un lado, un modelo de partido a la americana, llamado ligero, de carácter electoral, fuertemente identificado con la figura de líder y capaz de dirigirse a todo el país. Del otro, un modelo de partido a la europea, estructurado y pesado, entendido como evolución directa del modelo de partido de integración de masas, con una sólida organización territorial y un electorado de referencia preciso. Mientras que la secretaría de Veltroni abogaba por la primera opción, la segunda fue apoyada por importantes sectores de los dos grupos dirigentes de los partidos fundadores, con D'Alema (representado a los ex DS) y Marini (en representación de los ex DL) a la cabeza.

El modelo de partido ligero estaba llamado a encarnar un sujeto político de vocación mayoritaria, expresión duvergeriana que representaba la idea de un partido que aspira a conseguir un consenso electoral suficiente como para poder gobernar en solitario, apoyándose, para ello, en un liderazgo sólido, reconocido y orientado a conquistar un electorado amplio a través de un discurso no ideológico. El modelo de partido pesado, de otra parte, correspondía a la ambición de reconstruir un partido de integración de masas, capaz de representar principalmente a determinados sectores sociales y al mundo del trabajo (in primis, trabajadores públicos y asalariados). Un partido que solo podría presentar su candidatura al Gobierno del país a través de la construcción de una coalición con otras fuerzas políticas de centro y de izquierda. La victoria en las primarias de Veltroni favoreció la afirmación del primer modelo. Deduciendo de la lógica de vocación mayoritaria la identificación entre leadership y premiership, Veltroni desarrolló la campańa electoral de 2008 renunciando a cualquier posible alianza con otras fuerzas de la izquierda (Rifondazione Comunista, Verdi y otros exponentes de la izquierda radical bajo la bandera de Sinistra Arcobaleno) y aliándose, en exclusiva, con L'Italia dei Valori, del exmagistrado Antonio Di Pietro.

Las elecciones de 2008 vieron al PD situarse cerca del $33 \%$ (un consenso que con anterioridad solo había conseguido el PCI de Berlinguer en 1976), aunque el logro no fue suficiente para vencer al centro-derecha, representado por la coalición llamada Popolo della Libertà que lideraba Silvio Berlusconi ${ }^{11}$. El golpe de la derrota, a pesar del exitoso $33 \%$, fue sentido. Y la pérdida a los

11 El PdL era un nuevo partido liderador por Silvio Berlusconi, fundado en 2009 como resultado de la fusión de Forza Italia y Alleanza Nazionale, aunque participaría en las elecciones de 2008 como lista electoral. Una fusión que respondía al nacimiento del 
pocos meses de dos regiones importantes como Los Abruzos y Cerdeña obligaron a Veltroni a presentar su dimisión en febrero de 2009. Se hizo cargo del partido, tras ser elegido por la Asamblea Nacional, Dario Franceschini, que acompañó al partido hasta las elecciones europeas de 2009, en las cuales consiguió un consenso del $26 \%$, siete puntos menos que en las elecciones de 2008 . El camino hacia nuevas primarias, en todo caso, quedó abierto y en octubre de ese mismo ańo el partido celebró la elección de Pierluigi Bersani como nuevo secretario del PD. En el proceso de primarias participaron mas de tres millones de selectores, el $53 \%$ de los cuales votó por Bersani, mientras que el $34 \%$ dio su confianza a Franceschini y el $12,5 \%$ a Marino ${ }^{12}$.

\section{EL PARTIDO OLD STYLE DE BERSANI}

La victoria de Bersani en la batalla por la secretaría produjo una inversión en la tendencia organizativa del PD, orientándolo hacia un modelo de partido socialdemócrata old style. Los elementos diferenciales del nuevo partido fueron un peso mayor a la estructura organizativa, asociado a una mayor centralidad de los círculos, el desplazamiento del centro de gravedad del partido de los electores (party un public office) al grupo dirigente (party in central office), así como la asignación de un nuevo rol estratégico a la secretaría, convirtiéndola en el trampolín de entrada en el Parlamento. La imagen de la «ditta» (empresa o compañía), con la que el mismo Bersani bautizó su idea de partido, sintetizaba de manera eficaz la voluntad del nuevo grupo dirigente de reconstruir una comunidad política en torno a los valores tradicionales de la izquierda socialdemócrata, apoyándose en un sólido anclaje con los grupos sociales clásicos de referencia. Una elección que implicaba, de manera inevitable, el diseño de una estrategia de alianzas abierta a otras fuerzas políticas representativas de otros sectores, partiendo de un electorado moderado y de centro.

Algunos exparlamentarios de La Margherita, guiados por Francesco Rutelli, decidieron salir del PD y dar vida a Alleanza per l'Italia (API), señalando en la posición de Bersani una fuerte contradicción con las razones que habían llevado a la creación del nuevo sujeto político. A pesar de ello, en las elecciones municipales de 2011 el PD consiguió algunas victorias importantes — como en Milán, donde Giuliano Pisapia puso fin a veinte años de

PD y que fue anunciada en Milán el 18 de novimebre de 2007, en el famoso «discurso del predellino» de Berlusconi.

12 Con el término selectores (selettori) se hace referencia al conjunto de electores, formado por afiliados y simpatizantes, que participan como subgrupo en los procesos de primarias del PD para la elección del secretario del partido. 
administración del centro-derecha-, si bien no siempre con candidatos del partido a las alcaldías. En este proceso, el Gobierno de Berlusconi, cuya mayoría de gobierno no fue capaz de enfrentarse al riesgo del default que estaba tomando cuerpo a raíz de la crisis económico-financiera de 2008, entró en crisis. Fue sustituido a finales de 2011 por un Ejecutivo técnico liderado por Mario Monti y el apoyo externo del PdL y el $\mathrm{PD}^{13}$. Monti venía respaldado por su trayectoria como excomisario europeo y presidente de la Universidad Bocconi, y poco antes había sido nominado senador vitalicio por el presidente de la República Giorgio Napolitano.

A medida que el final de la legislatura se aproximaba, la estrategia de alianzas de Bersani se orientó hacia la construcción de una coalición con las fuerzas tradicionales del centro-izquierda, con las que ya se había aliado con ocasión de las elecciones administrativas de 2011, coalición que en el otoño de 2012 celebró primarias para la elección del candidato al Palazzo Chigi. El PD, de acuerdo con el principio de identificación entre premiership y leadership, debería haber puesto en liza solamente a su secretario, Bersani. Sin embargo, en el partido había crecido un frente de oposición bastante amplio, que apuntaba a la necesidad de eludir la alianza con las fuerzas políticas a la izquierda del PD para volver al modelo de partido de vocación mayoritaria de cuño veltroniano. Este frente, de orientación reformista liberal-democrática, vio una ventana de oportunidad para volver a la iniciativa política con el Gobierno Monti. Además, encontró en la figura del joven alcalde de Florencia, Matteo Renzi, un candidato a las primarias alternativo a Bersani. Gracias a una derogación excepcional del Estatuto del PD, apoyada por el mismo Bersani, Renzi presentó su candidatura a las primarias de la coalición junto al líder de SEL, Nichi Vendola, el representante del centro moderado Tabacci y Laura Pupato, consejera regional del PD en la región del Veneto. Las primarias Italia Bene Comune, organizadas con un mecanismo de doble turno, se celebraron el 25 de noviembre y el 2 de diciembre. Se saldaron con la participación de más de tres millones de votantes en el primer turno y algo más de 2800000 en la segunda vuelta. Venció Bersani, que en el balotaje obtuvo el $61 \%$ de los votos, contra un inesperado 39\% de los votos obtenido por Matteo Renzi. En la victoria de Bersani fue decisivo el apoyo de Vendola, quien invito a sus votantes, el 15,6\% en el primer turno, a apoyar al secretario del PD en el cargo.

13 El Gobierno de Mario Monti podía considerarse, a todos los efectos, un Gobierno técnico, dado que en su composición no figuraba ningún político. Desde el punto de vista parlamentario, además de PD y PdL, el Gobierno Monti gozaba del apoyo externo de otras fuerzas políticas como la API de Francesco Rutelli y el FLI de Gianfranco Fini, además de Italia dei Valori de Antonio Di Pietro, si bien este último partido decidió abandonar la mayoría el 16 de diciembre 2011. 
Las elecciones de 2013 llevaron al PD de Bersani a conseguir un resultado muy por debajo de las expectativas. El PD obtuvo solamente el 25,43\% de los votos a la Cámara de los Diputados, siendo el segundo por detrás del imprevisto éxito del M5S, el nuevo sujeto político nacido por iniciativa del cómico Beppe Grillo ${ }^{14}$. Gracias al resultado total de la coalición, el PD superaba los diez millones de votos y conseguía el premio a la mayoría en la Cámara. Sin embargo, en el Senado, donde el premio a la mayoría se otorga con base regional, los números fueron favorables a la oposición de centro-derecha, la cual, si bien había conseguido un punto porcentual menos de votos, obtenía 116 escaños, tres más que el centro-izquierda. Por otro lado, el PD perdía cerca de cuatro millones de electores en comparación con las elecciones de 2008.

La tripolarización del Parlamento, dividido entre el centro-izquierda, centro-derecha y el M5S, convertía al país en prácticamente ingobernable. En este contexto, Bersani se encontró con la dificultad de tener que gestionar una situación política inédita. Las negociaciones iniciadas con el M5S de Grillo para construir una mayoría de gobierno no llegaron a buen puerto. Por otro lado, en la elección del presidente de la República el PD se mostró incapaz de llevar al Palacio del Quirinal a ninguno de sus nombres, ni Franco Marini ni Romano Prodi. Aún más, sus candidaturas fueron víctima de francotiradores del proprio partido. De este modo, tras la reelección de Giorgio Napolitano como presidente de la República, Bersani dimitió. La secretaría del PD pasó a manos del exsecretario general del sindicato CGIL, Guglielmo Epifani. Mientras tanto, se puso en marcha el Gobierno de Enrico Letta, vicesecretario del PD desde 2009, quien lideró una gran coalición apoyada por fuerzas políticas de centro-derecha y centro-izquierda, principalmente el PD y el PdL. Este nuevo gobierno nacía bajo el signo de la continuidad en relación con el trabajo de saneamiento económico y financiero puesto en marcha por el anterior gobierno Monti, con el objetivo, incluso, de proceder a la reforma de la ley electoral y las instituciones para dar una mayor estabilidad al gobierno del país.

\section{EL PARTIDO PRAGMÁTICO DE RENZI}

La salida de la escena de Bersani y el horizonte de nuevas primarias a la secretaria del PD relanzó la figura de Renzi en la carrera por el liderazgo del

14 El M5S, gracias a la campańa electoral de Beppe Grillo, basada en el ataque incondicional a la clase política, consiguió ser el primero partido de la Cámara de los Diputados con el 25,56\%, mientras que en el Senado fue la segunda fuerza con el $23,79 \%$ de los votos, por detrás del PD, que consiguió el 27,43\%. Sin embargo, debe subrayarse que Beppe Grillo no fue candidato al Parlamento. 
partido. Un político que volvía a proponer con fuerza la idea del PD como sujeto político de vocación mayoritaria, pero llevando este modelo más allá de la concepción del partido que tenía Veltroni al identificar, para más señas, la idea del cambio político con la renovación radical de los grupos dirigentes del partido (que ilustró con el eslogan brutal de la «rottamazione», el desguace). Las primarias para la elección del nuevo secretario del 8 de diciembre llevaron a la participación a poco más de 2800000 selectores, por debajo de los tres millones que habían marcado las dos anteriores citas del PD. Renzi venció sin el apoyo de los viejos grupos dirigentes del partido, recogió el $67,5 \%$ del consenso y se destacó frente a sus competidores. Cuperlo, que encarnaba la línea política de continuidad con Bersani, solo alcanzó el 18,2\% de los votos, mientras que Civati, candidato outsider y expresión del ala libertaria y radical del partido, aquella ligada al movimentismo de izquierda, solo alcanzó el $14,2 \%$ de los votos.

El enfoque político de Renzi se caracterizó por un decidido pragmatismo. Dejando atrás la tradición ideológica de la izquierda, entendió el partido como un instrumento subordinado a la acción del líder y su objetivo primero: conquistar el Gobierno. En un breve lapso de tiempo, Renzi obligó al Gobierno Letta, cuya acción parecía demasiado condicionada por los vetos de las distintas partes de la coalición, a dimitir. Y el 17 de febrero Renzi culminó su ascenso al recibir el encargo de dar vida a un nuevo Ejecutivo, con el objetivo de acelerar las reformas económicas e institucionales que el Gobierno saliente ya tenía en su agenda.

El primer acto importante del Gobierno Renzi fue el llamado Pacto del Nazareno, un acuerdo con el centro-derecha de Silvio Berlusconi que recogía un principio de acuerdo para la reforma de la ley electoral, de la Constitución y de la elección del próximo Presidente de la República ${ }^{15}$. En el frente interno, de otra parte, Renzi se convirtió en el protagonista de una verdadera mutación genética del partido (Addario y Fasano, 2015). Una mutación que alejaba al PD de su ubicación ideológica original, fuertemente condicionada por valores que pertenecían a la tradición de la izquierda, con origen tanto en su componente postcomunista como en las aportaciones de la izquierda exdemocristiana.

Una de las características principales del nuevo estilo de liderazgo ejercido por Renzi era la búsqueda de una relación directa con los ciudadanos,

15 El acuerdo tuvo como consecuencia el retorno a la escena política de Silvio Berlusconi, condenado de manera definitiva a trabajos sociales por fraude fiscal. Su presencia en la sede del PD para firmar el acuerdo, registrado por prensa y televisión, constituía, desde un punto de vista simbólico así como político, el significado de una sustancial rehabilitación del líder del centro-derecha italiano. 
desarrollada a través de una campaña permanente que ponía en el centro de la opinión pública la acción cotidiana del jefe del Gobierno. La lógica de esta forma de entender el gobierno llevó a reducir el debate con los demás partidos de la mayoría y, en la arena partidista, a negar cualquier tipo de estrategia de concertación con las minorías internas ${ }^{16}$. En el campo electoral la primera reacción de la opinión pública fue positiva, como señalaba el $40 \%$ del consenso alcanzado en las elecciones europeas de $2014^{17}$. En la esfera gubernamental, la sustancial debilidad de sus socios de coalición hizo de la estrategia renziana una opción ganadora ${ }^{18}$. En el seno del PD, sin embargo, la impronta del liderazgo y el pragmatismo de Renzi comenzaron a generar una fuerte corriente de oposición entre las minorías. Algunos exponentes de relevancia (como Fassina, Civati, Mineo, D’Attorre o el estudioso Carlo Galli) decidieron abandonar el PD. Algunos confluyeron en Sinistra Ecologia Libertà (SEL), otros dieron vida a nuevos movimientos políticos, como Possibile o Sinistra Italiana. En todo caso, estos movimientos no lograron pacificar el clima interno del partido, dado que una componente importante del grupo dirigente postcomunista, encabezada por Pierluigi Bersani y Massimo D’Alema - que acogía en su seno a Gianni Cuperlo y Roberto Speranza, presidente de la Asamblea Nacional del partido y jefe del grupo parlamentario en la Cámara- decidió contrarrestar con fuerza las iniciativas de Renzi, ya fuera dentro del partido como en el debate político-parlamentario.

Algunos actos del Gobierno Renzi, como la reforma del mercado de trabajo y la consiguiente abolición del artículo 18 del Estatuto de los Trabajadores, asumieron la forma de una fuerte ruptura tanto con electorado de la izquierda como con algunas organizaciones tradicionalmente vinculadas al partido, comenzando por el sindicato $\mathrm{CGIL}^{19}$. Se iniciaba, de este modo, un lento pero inexorable proceso de desgaste de Renzi y su Ejecutivo, que sufría una

16 La distinción entre arena electoral, arena gubernamental y arena partidista se toma, en la perspectiva de un análisis inspirado en la lógica de la presidencialización de la política, de Potgunke y Webb (2006). Sobre el mismo tema para el caso italiano, véanse, además Calise (2006), Passarelli (2015), Musella (2015) y Musella y Webb (2015).

17 Un resultado cuya excepcionalidad se debe, en parte, a la presencia de un alto nivel de abstencionismo.

18 Resultaba cierto, con más razón, tras la ruptura del Pacto del Nazareno con Berlusconi, que redujo los aliados de centro-derecha solo al NCD, partido que nacía finales de 2013 como escisión del PdL por obra de parlamentarios que, bajo el liderazgo de Alfano, confirmaron su apoyo al Gobierno de Renzi.

19 El artículo 18 representaba una suerte de protección del despido improcedente y sin causa económica en empresas con más de quince empleados. 
aceleración después de la elección de Sergio Mattarella a la presidencia de la República. Una elección que, si bien de un lado permitió a Renzi acallar temporalmente las diferencias con la minoría crítica de su partido, del otro llevaba a la ruptura del Pacto del Nazareno sellado con Berlusconi. Por tanto, la trayectoria de Renzi como presidente del Consejo de Ministros se vio progresivamente asediada por dos tipos de oposición: una interna, desarrollada por iniciativa de la minoría crítica de su partido, la cual, a pesar de compartir la elección de Mattarella como presidente de la República, mantuvo una actitud firme de oposición ante las decisiones de Renzi y su Gobierno; y otra externa, originada por la voladura del pacto con el centro-derecha liderado por Berlusconi, el cual, en un principio, había permitido a Renzi aprobar una reforma de la ley electoral (introduciendo un sistema de doble vuelta y premio a la mayoría) y proceder al proyecto de revisión de la segunda parte de la Constitución de 1948. Los primeras señales que apuntaban a una pérdida de consenso del Gobierno de Renzi se manifestaron con ocasión de las elecciones administrativas de la primavera de 2016, cuando los candidatos del PD fueron derrotados en ciudades importantes —como Nápoles, Roma y Turín-, mientras que entre las grandes ciudades solamente Bolonia, Cagliari y Milán permanecieron en manos de mayorías del PD.

Ante la progresiva dificultad para gobernar, Matteo Renzi optó por relanzar el desafío a sus opositores vinculando su propia suerte, como la del Gobierno, al resultado del referéndum constitucional. El 4 de diciembre de 2016 el voto contrario a la reforma constitucional acarició el $60 \%$, con una participación que llegaba casi al $70 \%$. Un resultado que llevaba impresa la huella del gran frente de oposición que se organizó contra el presidente del Consejo de Ministros, en el que cabían desde los partidos de la oposición al Gobierno, la minoría del PD encabezada por D’Alema y Bersani y organizaciones de la órbita de la izquierda italiana como el sindicato CGIL o la asociación de partisanos ANPI. Tras las derrota, Renzi presentó su dimisión como presidente del Consejo de Ministros, dejando paso a Paolo Gentiloni, ministro de Exteriores de su Ejecutivo. Un par de meses después, en febrero de 2017, Renzi también dimitió como secretario del $\mathrm{PD}$ y puso en marcha el proceso para la celebración de nuevas primarias para la elección del secretario del partido ${ }^{20}$.

El nombramiento del Gobierno Gentiloni permitió agotar la XVII legislatura de manera natural. En este tiempo, Matteo Renzi anunció la decisión

20 En el interregno entre la dimisión de Renzi como secretario del partido en febrero de 2019 y la reelección del secretario el 30 de abril del mismo año, el liderazgo del PD fue asignado, en calidad de regente ad interim, al presidente de la Asamblea Nacional, Matteo Orfini. 
de presentar nuevamente su candidatura a dirigir el PD. Su movimiento provocó la escisión de la minoría socialdemócrata guiada por Bersani, D’Alema y Speranza, grupo que dio vida a una nueva formación política, Articolo 1-Movimiento Democratico e Pogressista, en el que confluyeron una treintena de parlamentarios del PD y una veintena de parlamentarios de SEL. La primarias del PD tuvieron lugar el 30 de abril de 2017, y registraron un descenso importante de la participación, con casi un millón y medio de selectores menos que en 2013. Renzi fue reelegido para dirigir el partido con el $70 \%$ de los votos, contra el $20 \%$ conquistado por el ministro de Justicia Andrea Orlando y el $10 \%$ de los votos obtenidos por el gobernador de la Puglia, Michele Emiliano. Las primarias de 2017 mostraron que Renzi había consolidado un gran consenso entre los selectores del PD. Sin embargo, su segundo mandato al frente del partido iba a ser una lenta y resignada marcha hacia las elecciones de 2018, las cuales casi todos en el PD daban por perdidas.

Desgarrado por profundas divisiones internas, el centro-izquierda se presentó a las elecciones de 2018 fragmentado en distintas listas que se hacían la competencia. Además del PD, concurrieron a las elecciones dos nuevas formaciones políticas de izquierda - Liberi e Uguali (en el cual se reencontraron Articolo 1, SEL y Possibile, de Civati) y Potere al Popolo. Los resultados de las elecciones de 2018 vieron la neta afirmación del M5S como partido más votado, apoyado por casi un tercio de los votantes. El PD sufrió un claro retroceso al certificar un decepcionante $18,7 \%$ de los votos en la Cámara de los Diputados, mientras que en el Senado no lograba ir más allá del 19\%. Una derrota que provocó una nueva dimisión de Renzi como secretario del PD, dimisión que se producía en medio de la desaprobación pública, incluso por parte de un amplio sector del grupo dirigente, de la orientación pragmática que el liderazgo de Renzi había impreso al partido.

La Asamblea Nacional del PD eligió como secretario regente al exministro de agricultura Maurizio Martina, quien asumió la tarea de guiar al partido hacia un nuevo proceso de primarias, convocadas para el 3 de marzo de 2019. De este modo, a poco más de diez años del nacimiento del partido, el PD se encontró atravesando una de las fases más complicadas de su breve historia. Los miembros del PD vinculados al proyecto y liderazgo de Renzi fueron protagonistas de una verdadera diáspora, viéndose obligados a elegir entre los tres candidatos en liza: el gobernador de la región del Lazio, Nicola Zingaretti; el secretario regente Martina o el exradical Roberto Giachetti. En cualquier caso, el juicio y valoración que merecía el liderazgo renziano marcó el debate congresual, dividiendo el partido entre quienes reivindicaban con orgullo el periodo renziano - grupo compuesto por una exigua minoría que se reconocía en la candidatura de Giachetti- y quienes consideraban 
necesario marcar distancias con la experiencia del renzismo, como Zingaretti $y$, en parte, Martina ${ }^{21}$.

\section{EL PARTIDO OMNIBUS DE ZINGARETTI}

Las primarias del 3 de marzo de 2019 se celebraron en un clima de relativa indiferencia por parte de los ciudadanos y la opinión pública, y registraron una participación de en torno a 1580000 selectores, dos tercios de los cuales votó por Zingaretti, mientras que Martina y Giachetti obtuvieron, respectivamente, el $22 \%$ y el $12 \%$ de las preferencias. Con la victoria de Nicola Zingaretti se afirmaba, sobre todo en la mayoría que formaba el nuevo grupo dirigente, la voluntad de cerrar una etapa de la historia del PD marcada por la centralidad del líder. A tal efecto, el Estatuto del partido fue modificado, eliminando la norma que identificaba leadership y premiership. Dicho de otro modo, deshaciendo el principio que convertía al secretario del partido de manera automática en el candidato a liderar la coalición del centro-izquierda a las elecciones ${ }^{22}$. El estilo de liderazgo más colegial e inclusivo desplegado por Zingaretti, acompañado de una disposición constructiva por parte de la minoría liderada por Martina, se tradujo en una reducción efectiva de la conflictividad interna del partido. Si bien aún permaneció abierto el frente con el grupo representado por Giachetti, cuya acción se centraba en poner de manifiesto las diferencias con el espíritu del nuevo liderazgo, más cercano al proyecto representado por Bersani que a la inspiración reformista y pragmática del estilo de gestión renziano. La acción estratégica de Zingaretti, en todo caso, estuvo orientada desde el inicio a colmar las expectativas de quienes interpretaron el resultado de las primarias como una demanda de mayor unidad en el partido, tanto en el seno del grupo dirigente como en las bases territoriales. Una cohesión que el nuevo líder puso en el centro de la agenda en detrimento, incluso, de la visibilidad mediática y eficacia de su liderazgo.

Zingaretti tuvo que enfrentarse a la prueba de las elecciones al Parlamento Europeo celebradas en junio de 2020, si bien en el partido prevaleció la predisposición a no hacer de su primer lance electoral un juicio sobre el

21 Para comprender el calado y dimensión de la diáspora de los apoyos de Renzi, y hasta qué punto fue controvertido el juicio sobre la experiencia renziana incluso para quienes fueron participes, puede observarse que entre los partidarios de la candidatura de Zingaretti estaba el expresidente del Consejo de Ministros Paolo Gentiloni, cuya acción de gobierno se desarrolló bajo el signo de la continuidad con el Gobierno Renzi.

22 Véase el nuevo Estatuto del PD, aprobado por la Asamblea Nacional el 17 novembre 2019: https://www.partitodemocratico.it/statuto/. 
naciente liderazgo. En todo caso, la sensible recuperación del PD, que obtuvo el $22,74 \%$ de los votos, cuatro puntos más que en las elecciones italianas de 2018, fue acogida de manera positiva. El resultado de las elecciones europeas en Italia contribuyó a agudizar la fase de crisis y conflicto abierta entre los miembros del Gobierno de coalición formado por la Lega y el M5S en 2018 ${ }^{23}$, coyuntura que brindó al PD la posibilidad de adquirir una nueva e inesperada centralidad en la política italiana. Con la retirada unilateral de la Lega del Gobierno, el Ejecutivo de Conte fue privado de apoyo parlamentario y, a pesar de la fuerte presión ejercida por Renzi sobre el nuevo grupo dirigente del partido, en septiembre de 2019 el PD decidió dar vida a un nuevo Gobierno de coalición con el M5S, con Giuseppe Conte, de nuevo, como presidente del Consejo de Ministros. De este modo, el segundo Gobierno de Conte llevaba de nuevo al PD a la dirección del país. Sin embargo, entre los motivos que llevaron al PD al Gobierno de la mano del M5S pesó más el miedo a un retorno prematuro a las urnas (donde los sondeos reflejaban una Lega de Salvini en crecimiento constante, a punto de alcanzar el $40 \%$ del consenso), que la voluntad de imprimir un giro de ciento ochenta grados al Gobierno. Dos semanas después del nacimiento del nuevo Gobierno, Matteo Renzi anunció su salida del PD, junto a veintinueve diputados (incluido Giachetti) y dieciséis senadores, para dar vida a un nuevo partido: Italia Viva, si bien, de momento, decidía mantener su apoyo al Ejecutivo Conte II.

Llegamos, de este modo, al presente, con un PD comprometido en una difícil acción de gobierno al lado de un socio de coalición audaz, pero privado de orientación política, y con una opinión pública que no parece premiar el hecho de que el PD se haya hecho cargo del Ejecutivo, dado que los sondeos continúan señalando como opción preferente a la Lega de Salvini y, en general, la coalición de centro-derecha con Forza Italia y Fratelli di Italia. En este sentido, las elecciones en la región de la Umbría, celebradas en octubre de 2019, reflejaban esta tendencia con una derrota histórica del PD (en coalición con el M5S), que fue superado por la Lega como primer partido en la región, un feudo tradicional de la izquierda italiana. Una victoria que el líder de la Lega, Matteo Salvini, instrumentalizó para desafiar al PD y al Gobierno, en general, señalando que si el centro-derecha vencía en las elecciones de la

23 En las elecciones al Parlamento Europeo del 26 de mayo de 2019, la Lega de Matteo Salvini obtuvo el 34,3\% de los votos, mientras que el M5S consiguió un decepcionante $17 \%$. Un resultado que cambiaba los papeles de ambos partidos respecto a las elecciones italianas de 2018, en las que el M5S obtuvo el 32,7\% del consenso y el partido de Salvini el $17,4 \%$, lo que contribuyó, en buena medida, a acentuar el conflicto entre los dos partidos. 
Emilia-Romagna el Ejecutivo Conte debería dimitir. Sin embargo, si bien los sondeos negaban la victoria al PD y la coalición de izquierda (esta vez sin el M5S), la movilización política organizada por el naciente movimiento llamado las Sardinas llevaron a la victoria al candidato del PD Stefano Bonaccini.

Apoyándose en esta victoria y la obtenida en Calabria, que permitían al partido tomar aire, Zingaretti anunció una nueva fase política guiada por la necesidad de construir un "partido nuevo»" ${ }^{24}$. Una expresión de inspiración togliattiana con la que el secretario del PD buscaba subrayar la voluntad de abrir el partido a distintas fuerzas de la sociedad civil — como, por ejemplo, la que en los últimos meses habían representado las Sardinas - para convertirse en el centro de una nueva y amplia alianza de centro-izquierda capaz de encarnar una alternativa creíble al centro-derecha liderado por Salvini. Una suerte de nuevo inicio que, teniendo poco que ver con los liderazgos precedentes $^{25}$, avanza la forma de un partido ómnibus en el que pueden coexistir orientaciones políticas diferenciadas, incluso profundamente distintas, pero a riesgo de permanecer unidas no tanto por le hecho de haber encontrado algún tipo de síntesis, sino por razones de mera conveniencia política y electoral.

\section{EVOLUCIÓN DEL CONSENSO ELECTORAL DEL PD}

La evolución del consenso electoral del PD, verdadera cara y cruz del partido, muestra una trayectoria inconsistente a lo largo del tiempo. Tras un inicio positivo, que culmina con el 33,2\% de los votos en las elecciones de 2008, la capacidad de atracción del partido para el votante italiano sufre un lento e inexorable declive. Incluso el resultado de 2008, si se analiza bien, como muestra el gráfico 1, está vinculado a los votos que DS y La Margherita obtuvieron en las dos elecciones precedentes, con listas separadas en 2001 y unidos en Uniti nell'Ulivo del 2006. Por el contrario, el PD registra su consenso más bajo con ocasión de las últimas elecciones celebradas en 2018, un $18,7 \%$, después de la experiencia gubernamental del PD con Renzi y Gentiloni al frente. La única excepción, si bien favorecida por un alto nivel de

24 Entrevista de Zingaretti a La Repubblica, 11-06-2020, pp. 2-3.

25 La misma comparación con el PD en su versión bersaniana, aparentemente más próxima a la idea de partido de Zingaretti, no puede considerarse suficientemente fundada, dado el reequilibrio de fuerzas que el injerto de nativos había producido en el caso zingarettiano, respecto a la preponderante presencia de exmiembros del DS - cuando no, por ańadidura, ex del PCI - que diferenciaba a la era bersaniana (Fasano y Natale, 2019). 
abstencionismo, la constituye el voto al Parlamento Europeo de 2014, que premiaba el naciente liderazgo de Matteo Renzi, instalado ya en el Palazzo Chigi desde febrero de ese año. Tras las últimas elecciones europeas de 2019, testigo de una recuperación, si bien contenida, del partido respecto a las elecciones italianas de 2018, podemos afirmar que el PD, a nivel nacional, todavía no ha consolidado un umbral equiparable a un cuarto del electorado italiano. Un consenso electoral muy por debajo de las expectativas alimentadas en la fase constituyente del partido por los dos partidos fundadores, que a comienzos de la década pasada superaban el $30 \%$ de los votos en su conjunto.

Gráfico 1. Voto a DS y DL-La Margherita a la Cámara de los Diputados (2001 y 2006). Voto al PD a la Cámara de los Diputados (2008, 2013, 2018) y al Parlamento Europeo (2009, 2014, 2019)

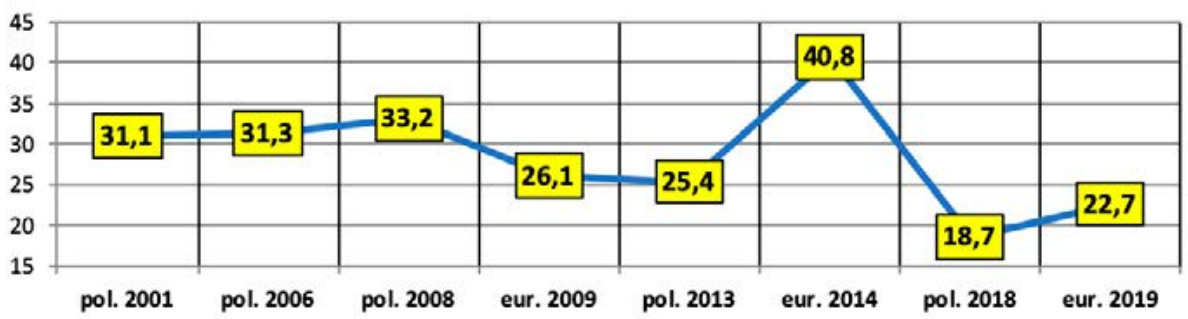

Fuente: elaboración propia.

Los estudios demoscópicos nos dicen que esta evolución se ha visto acompañada de algunos momentos de elevado consenso (Natale y Fasano, 2017). Por ejemplo, en coincidencia con la dimisión del Gobierno Berlusconi en noviembre de 2011 y la investidura del Gobierno técnico guiado por Mario Monti, así como en los meses precedentes a las elecciones de 2013, numerosos sondeos estimaban al PD entorno al $30 \%{ }^{26}$, si bien la respuesta efectiva de las urnas, por debajo de las expectativas maduradas a lo largo de la campaña electoral, se tradujo en un decepcionante $25 \%$, un resultado que acusaba el inesperado éxito del M5S, cuya irrupción ilustraba de manera clara todas las dificultades del partido para convertirse en un punto de referencia sólido para el electorado. De igual modo, en los primeros dos años con Renzi al frente, el PD volvió a igualar los niveles de consenso que habían distinguido al partido en sus inicios, hasta superarlos, incluso, con el resultado de más del $40 \%$

26 Para tal propósito, véase Natale y Fasano (2017: 135-137). 
obtenido en las elecciones europeas de 2014. Sin embargo, en la segunda fase del liderazgo renziano, tras la derrota en el referéndum constitucional del 2016, el consenso entorno al PD comenzó a debilitarse, hasta tocar suelo con el $18,7 \%$ de las elecciones de 2018 .

La falta de continuidad en la evolución electoral del PD se explica, sobre todo, atendiendo a dos razones. Primero, las profundas divisiones que han atravesado la izquierda italiana, tanto en el seno del PD como en todo el campo de las fuerzas del centro-izquierda. Segundo, la profunda transformación del sistema de partidos italiano que se registra en la última década, sobre todo debido a la irrupción de un partido antisistema como el M5S (Biorcio y Natale, 2013; Corbetta y Gualmini, 2013: Chiapponi, 2017) y la mutación de la Lega de partido federalista a soberanista (Passarelli y Tuorto, 2018).

\section{UN PARTIDO DIVIDIDO: TRES ALMAS DISTINTAS Y UN CONFLICTO IRRESOLUBLE}

La rápida sucesión, en el marco de una docena de años, de siete secretarios y cinco asambleas y direcciones nacionales, es una primera seńal que evidencia la inestabilidad permanente de los grupos dirigentes que han estado al frente del PD. Sin embargo, para comprender en qué medida las divisiones internas del partido han contribuido a perjudicar la consolidación de la organización es necesario atender a la configuración que han ido adoptando, en el curso del tiempo, la Asamblea y la Dirección Nacional ${ }^{27}$, recordando que

27 El estudio del cuadro militante activo, con particular atención a los delegados nacionales, constituye uno de los aspectos cruciales y neurálgicos de la vida organizativa y política de un partido. Como han puesto de manifiesto Reif et al. (1980), es considerada la verdadera estructura portante de los partidos, la middle-level party elite que se ubica entre el nivel local y el nacional, desarrollando importantes funciones de coordinación entre los diversos ámbitos organizativos que alimentan la vida cotidiana del sujeto político. Los delegados de los congresos nacionales (que, en el caso del PD, se corresponden con los miembros de la Asamblea Nacional) proporcionan una estampa fiel y representativa de este personal político, que por dedicación y implicación pueden considerarse, con razón, la verdadera columna vertebral del partido, cuando no el grupo de referencia para la selección de dirigentes y candidatos a distintos niveles (Bordandini et al., 2011; Bordandini y Cartocci, 2011; Raniolo, 2011). Sobre todo en una fase como la actual, en la que la mayor parte de los casos el party in central office, es decir, los dirigentes nacionales (que, en el caso del PD, se corresponden con los miembros de la Dirección Nacional), se solapan cada vez más con los representantes parlamentarios. Una primera contribución de importancia al estudio de los delegados 
ambos organismos - el primero elegido directamente y en paralelo a la elección del secretario, y el segundo (hasta el año pasado) aprobado por la Asamblea Nacional- se componen de acuerdo con el pluralismo congresual ${ }^{28}$. Por tanto, su composición refleja la presencia de las diversas componentes o facciones del partido, en tanto que se encuentran vinculadas a las listas de los candidatos que se presentan a las primarias.

La primera Asamblea Nacional, investida en el acto de constitución del PD, se caracterizó por una amplia presencia de mujeres y jóvenes: las primeras favorecidas por la composición de las listas y la adopción de la preferencia de género, los segundos como resultado de una decisión compartida por Veltroni y los grupos dirigentes de los dos partidos fundadores, a fin de asegurar para el nuevo sujeto político una imagen de renovación (Fasano, 2009; Natale y Fasano, 2017). Sin embargo, la naturaleza de merger party que caracterizaba al $\mathrm{PD}$, fruto de la fusión fría a la que nos hemos referido, encontró pronto una correspondencia en la orientación política del cuadro militante activo, dado que la Asamblea Nacional nacía con la fuerte impronta de exmiembros del DS y La Margherita. En todo caso, el liderazgo de Veltroni, capaz de combinar reformismo y radicalismo dentro de la lógica de un partido de vocación mayoritaria, junto a la voluntad de cohesión que acompañó a la fase constituyente del PD, contribuyeron de manera determinante a frenar la potencial conflictividad latente en el partido. Sobre todo en lo referente al campo de las cuestiones éticamente sensibles y de las reformas económicas y sociales (Natale y Fasano, 2017). La primera Dirección Nacional, sin embargo, se caracterizó por una marcada parlamentarización, un aspecto que señalaba una fuerte discontinuidad con las tradiciones de DS y La Margherita. Se trataba de una elección particular de Veltroni, quien aspirando a realizar un modelo de partido ligero y apoyado en los electores, fuertemente identificados con el líder, hizo del "party in public office» (Natale y Fasano, 2017) la columna vertebral de la organización.

La llegada de Bersani, elegido nuevo secretario del PD en 2009, produjo una radical inversión de tendencia. En primer lugar, la cuota juvenil sufrió una evidente contracción. Aumentaron, viceversa, los mayores de setenta años,

en los congresos nacionales de los partidos italianos, en una perspectiva diacrónica dedicada a examen la transformación de los principales partidos de la izquierda — primero el PCI, después el PdS y DS— es Bellucci et al.(2000). Para un análisis más reciente, con capacidad para considerar un número de partidos más amplio (PRC, DS, Margherita, UdC, Forza Italia y AN), véase Ignazi y Bordandini (2018).

28 En noviembre de 2019 fue aprobado un nuevo Estatuto del PD, en base al cual la Dirección Nacional del partido consta de 124 miembros. Para su composición y mecanismo de selección, véase https://www.partitodemocratico.it/statuto/. 
casi hasta una quinta parte de los delegados nacionales, muchos de los cuales, de otra parte, podían presumir de experiencia política en las filas del PCI o la DC (Fasano, 2010; Natale y Fasano, 2017). En segundo lugar, la lógica de la vocación mayoritaria fue archivada en favor de una estrategia de alianzas que, en todo caso, nacía bajo el signo de la indefinición. Si bien en un principio, Bersani advirtió como prioritaria la búsqueda de acuerdos con las fuerzas políticas de centro, al acercarse las elecciones de 2013 optó por la construcción de una coalición, Italia Bene Comune, en el que el protagonismo recayó sobre los partidos que se situaban a la izquierda del PD. En tercer lugar, la vinculación del proyecto de Bersani a un modelo de partido tradicional, de cuño socialdemócrata, terminó por acentuar las divergencias de fondo entre laicos y católicos dentro de la organización (Fasano, 2010; Natale y Fasano, 2017), diferencias que se reprodujeron, de manera más acentuada, en el seno de la Dirección Nacional, que estaba compuesta, sobre todo, por funcionarios de partido cuyo origen echa raíces en DS o La Margherita, contribuyendo, en definitiva, a que el enfrentamiento entre ambas partes sobre cuestiones éticamente sensibles y reformas económicas o sociales asumiese la connotación de un desencuentro no negociable (Natale y Fasano, 2017).

Con la nueva secretaría de Matteo Renzi, nacida bajo el signo de una renovación radical de los grupos dirigentes — como, de otra parte, daba a entender el eslogan «rottamazione»—, el PD experimentó un proceso de renovación y apertura hacia la sociedad civil. La composición de la Asamblea Nacional de 2013 mostraba esta cambio: por primera vez la presencia de delegados nativos del PD —es decir, aquellos que carecían de experiencia política previa en DS o La Margherita - era superior a la de quienes provenían de los dos partidos fundadores (Fasano y Martocchia Diodati, 2014a) ${ }^{29}$, dato que resultaría determinante a la hora de ofrecer al nuevo secretario un margen de acción discrecional desconocido por sus predecesores en el cargo. Sólidamente anclados en posiciones de centro-izquierda, desencantados frente a las tradiciones políticas europeas (aunque sin dificultad para definirse como

29 Se debe observar que con ocasión de la reelección del secretario, registrada en 2017, la componente de los nativos del PD fue reequilibrada por una igual presencia de delegados con origen en DS, sumando entre ambos en torno al $40 \%$ de la Asamblea Nacional (Fasano et al., 2018). Este hecho representaba un evidente paso atrás respecto a la lógica de la «rottamazione» que había prevalecido en la composición de la platea de delegados nacionales que acompańaron el primer mandato de Renzi. En cualquier caso, a partir de 2013 se registra una presencia consistente de nativos del PD, que más allá de su relativa superioridad numérica, iba a constituir un dato permanente del cuadro militante activo del partido. A tal propósito, véase también Natale y Fasano (2017). 
socialdemócratas de manera pragmática), abiertos a la presencia de la Iglesia en el debate público, al tiempo que favorables a cuestiones como los matrimonios del mismo sexo o la eutanasia, los nativos del PD parecían representar un apoyo ideal para la obra de renovación del partido que se había impuesto Matteo Renzi (Fasano y Martocchia Diodati, 2014a). En este sentido, la actitud que mejor define al nuevo grupo dirigente del PD era una orientación pragmática, más orientada a la solución de problemas viables que a la satisfacción de las expectativas de los grupos sociales vinculados tradicionalmente a la izquierda. En el ámbito de la Dirección Nacional, los nativos llegaron a alcanzar la cuota de un tercio, mientras que la presencia de los miembros elegidos superó a la de los funcionarios de partido, en coherencia con la idea que debía convertir a la acción de gobierno en la guía de la iniciativa política del partido (Natale y Fasano, 2017).

Tras los dos mandatos de Renzi, la victoria de Zingaretti, al menos desde el punto de vista de los grupos dirigentes y el cuadro militante activo del partido, nacía bajo el signo de la búsqueda de tranquilidad (Natale, 2019). Después de la sonora derrota que el partido encajó en las elecciones de 2018, los electores e inscritos del PD esperaban que el nuevo grupo dirigente fuese capaz de relanzar el partido, que desde la cita con las urnas parecía una organización subalterna a las fuerzas políticas en el Gobierno. En este contexto, la orientación general de la Asamblea Nacional, más que a la recuperación de los valores fundamentales del PD, como había evocado el nuevo secretario, parecía acercarse a la restauración del modelo de partido de la etapa de Bersani, volviendo a proponer un modelo de alternancia entre el alma socialdemócrata y la democrático-reformista (Natale, 2019). La presencia de jóvenes, como de los delegados entre treinta y cuarenta años, aumentó al tiempo que también aumentó el grupo de mayores de setenta años. Los llamados nativos, con más del $40 \%$ de los delegados, representaban la componente más numerosa de la Asamblea Nacional, seguidos, de cerca, por el grupo de delegados exmiembros del DS, que constituía el $36 \%$, mientras que el grupo de los delegados con origen en La Margherita aparecía en franco declive. En particular, resulta ilustrativo que con el nuevo liderazgo de Zingaretti, el partido volvía a movilizar a los postcomunistas dentro del cuadro militante activo del partido (Natale, 2019). El $70 \%$ de los delegados se definía como de centro-izquierda, si bien se registraba un aumento de quienes se definían exclusivamente de izquierda, hasta el $15 \%$, dentro de los cuales se encontraba, de manera significativa, el grupo de apoyo del nuevo secretario

Finalmente, la secretaría inaugurada por Zingeretti introduce un cambio decisivo en lo que respecta a la visión del partido, el liderazgo y su relación: cerca de dos tercios de la Asamblea considera que el deber principal del secretario es consolidar el PD, su perfil identitario, su línea política y su programa. 
Una diferencia sustancial con el periodo renziano, en el que la prioridad de la acción del líder del partido era ganar las elecciones y conquistar el Gobierno. La voluntad de dejar atrás la etapa del PD como partido basado en la centralidad del líder encontraba su reflejo en la marcada preferencia de los delegados, un $80 \%$, a favor del mantenimiento de una forma de gobierno parlamentario, redoblando desde el punto de vista porcentual el valor respecto a la etapa en la que el partido era guiado por Renzi (Natale, 2019).

Estas divisiones internas del PD se refieren, también, a diferencias de fondo sobre valores fundamentales ${ }^{30}$. Como es sabido, si en el mismo partido se encuentran grupos o facciones caracterizados por profundas diferencias en el plano de los valores, que se reflejan en la articulación de orientaciones políticas divergentes y producen iniciativas de signo diverso, resulta difícil que el partido pueda concebirse y representarse como un sujeto capaz de sostener una acción unitaria. Al mismo tiempo, es muy probable que los miembros de la organización privilegien su pertenencia de grupo o facción frente a la de partido.

Si comparamos las puntuaciones asignadas (en una escala del 1 al 10) a algunos valores fundamentales por parte de los delegados de la Asamblea Nacional del PD en 2009, 2013, 2017 y 2019, podemos observar que un núcleo de estos valores - tales como igualdad, paridad de oportunidades, la laicidad, el pacifismo, el medioambiente, el trabajo-, que son por su propia naturaleza universales y se refieren a una dimensión de autotrascendencia, presentan valores medios relativamente altos, entre el 8 y el 9, en los distintos grupos nacionales. En cambio, valores como el mercado y la competición, que se refieren a una dimensión de autoafirmación, se caracterizan por valores medios significativamente más bajos, entre el 6 y el 7, aunque estos últimos manifiestan un sensible crecimiento entre los delegados nacionales de 2013 y 2017, coincidiendo con los dos mandatos de Renzi, sobre todo entre los elegidos en la moción del secretario ${ }^{31}$. De este modo, en la transición de la Asamblea Nacional de 2009 a la de 2013, al conjunto de las referencias normativas compartidas por gran parte de los delegados, definidas en relación con valores universales ligados a la dimensión de autotrascendencia, como resulta de la tradición y horizonte ideológico y cultural en el que se encuadra la

30 Recordamos con Schwartz (1992, 1994) que los valores son convicciones, relativamente estables, sobre modos ideales y deseables de ser o actuar, fines motivacionales que guían el pensamiento y el comportamiento en distintos ámbitos de la vida.

31 Tomamos como referencia la tipología de valores de Schwartz (1992), quien distingue, con base en dos parejas de oposición, entre orientación a la conservación o el cambio y orientación universalista (autotrascendente) o de autoafirmación. 
izquierda, se suma un grupo de valores en buena medida extrańos a esta tradición, vinculados, por su naturaleza, a la dimensión de autoafirmación, como competición y mercado. Un injerto con consecuencias de fondo sobre el cuadro militante activo del partido, en la medida en que genera estímulos políticos que divergen progresivamente. Esta transformación del sistema de valores del PD se muestra con claridad en los gráficos 2, 3, 4 y 5, que ilustran en forma poligonal la puntuación media asignada a los diversos valores por parte de los delegados de las cuatro asambleas nacionales, ya sea en su conjunto como en relación a cada moción particular. Como puede observarse en la progresiva desalineación de los lados del polígono, a partir de la Asamblea Nacional de 2013, el sistema de referencias normativas de los delegados nacionales se va diferenciando de tal modo que parece sustancialmente irreversible (Fasano et al., 2018).

Gráfico 2. Valores fundamentales de los delegados de la Asamblea Nacional 2009

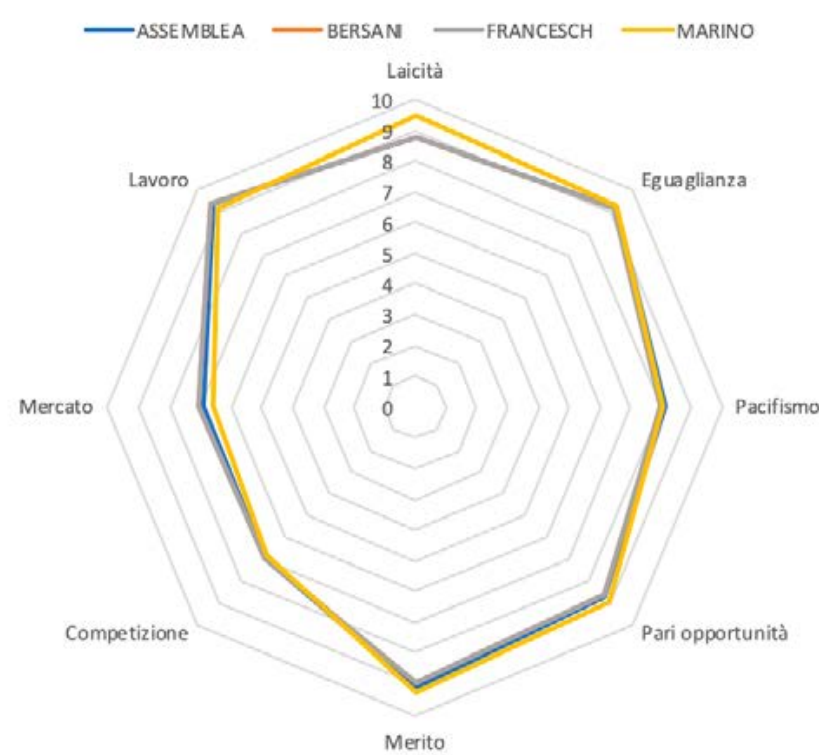

Fuente: elaboración propia. 
Gráfico 3. Valores fundamentales de los delegados de la Asamblea Nacional 2013

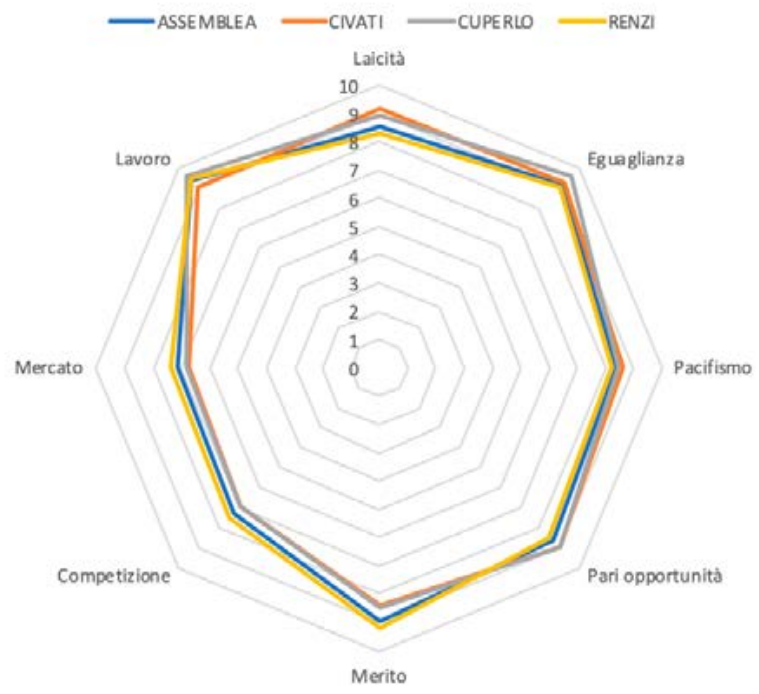

Fuente: elaboración propia.

Gráfico 4. Valores fundamentales de los delegados de la Asamblea Nacional 2017

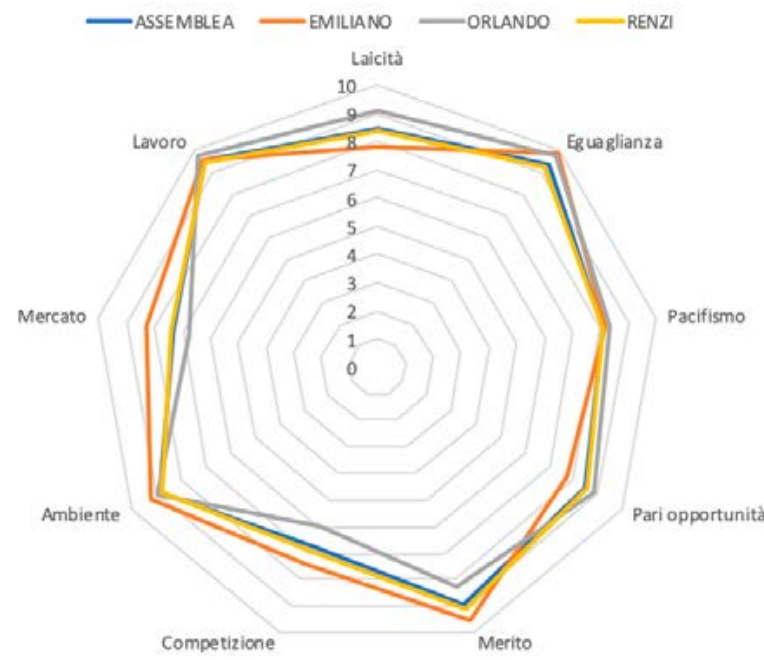

Fuente: elaboración propia. 
Gráfico 5. Valores fundamentales de los delegados de la Asamblea Nacional 2019

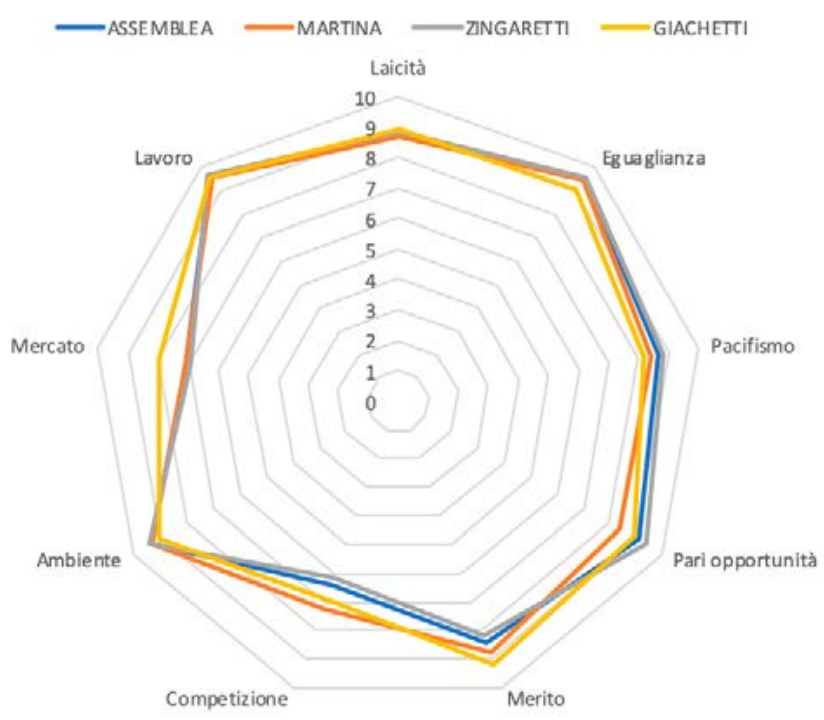

Fuente: elaboración propia.

Tampoco el recambio generacional, representado por el ingreso en el partido de nativos del PD, en función de cuotas numéricamente cada vez más consistentes, ha contribuido a limitar el grado de fragmentación experimentado por el sistema de valores del cuadro militante activo del partido. En ausencia de un compromiso claro por parte de los líderes o grupos dirigentes a la hora de construir una sólida y renovada cultura política, el resultado más obvio de la permanencia de dichas divisiones en el PD, ante todo de valores y después políticas, no ha sido otro que la dificultad del partido para asumir posiciones coherentes y unitarias frente a las cuestiones políticas importantes. Un problema que se proyecta sin solución, toda vez que el nuevo secretario y su grupo dirigente hasta ahora no parecen capaces de delinear una estrategia eficaz para refundar el partido sobre los presupuestos que habían motivado el nacimiento del PD.

\section{AFILIADOS Y SELECTORES}

Como se ha visto, el PD es un partido que nace bajo el signo de las primarias. Mejor dicho, de un procedimiento de elección directa del 
secretario, considerado a un tiempo el candidato del partido al cargo de Presidente del Consejo de Ministros, abierto a la participación de los electores. En la docena de años que han pasado desde su constitución se han celebrado cinco consultas de este tipo: 2007, 2009, 2013, 2017 y, finalmente, en 2019. A estos procesos deben sumarse las primarias celebradas para la selección del candidato a premier del centro-izquierda de 2012, con la participación, además del secretario del partido del momento, Pierluigi Bersani, de Matteo Renzi y Laura Puppato (Gelli, Mannarini y Talò, 2013). Todas estas elecciones se han caracterizado por un alto nivel de participación, a pesar de que desde las primarias que llevaron a la secretaria a Veltroni en 2007 a las últimas, que han sido testigo de la elección de Zingaretti como secretario del PD, el número de los selectores (léase, de los electores y afiliados que participan en las primarias) ha decrecido a ritmo constante (Natale y Fasano, 2017). Las primarias celebradas en 2007 y 2009 superaron el umbral de los tres millones de votantes, así como sucedió, al menos en el primer turno, de las primarias de la coalición Italia Bene Comune de 2012. Las primarias de 2013, sin embargo, movilizaron a poco más de 2800000 selectores, mientras que en 2017 y, finalmente, en 2019, la caída de la participación ha sido más que evidente, movilizando a menos de dos millones de votantes ${ }^{32}$.

Como puede observarse en el gráfico 6, la evolución de la tasa de participación en las primarias para la selección del secretario del partido (estimado en referencia a los votos obtenidos por el PD en las elecciones precedentes a la Cámara de los Diputados) ha oscilado, en el curso del tiempo, entre el $25 \%$ y el $32 \%$ (Natale y Fasano, 2017; Emanuele y Marino, 2019). Desde un punto de vista sociodemográfico, los selectores del PD presentan un perfil parecido al de su electorado. Se trata, principalmente, de un electorado de edad avanzada (más de un $65 \%$ supera los 45 años), con un nivel de educación relativamente alto (una mayoría clara presenta un título superior o licenciatura), trabajador asalariado y con una presencia notable de pensionistas (Natale y Fasano, 2017). Se trata, por tanto, de un selectorado que puede definirse como leal al partido per se, en tanto que tiende a sostener al vencedor de la consulta, aunque no se trate del candidato elegido. A pesar de ello, debe hacerse notar que en el curso del tiempo la cuota de los llamados votantes inciertos -es decir, aquellos que en caso de resultar derrotado su candidato no se sienten vinculados por el PD o la coalición de centro-izquierda — se ha incrementado sensiblemente (Cavataio y Fasano, 2014; Natale y Fasano, 2017).

32 Para un análisis detallado de las primarias del PD para la elección del secretario del partido, véanse Pasquino (2009), Pasquino y Venturino (2010 e 2014), Sandri y Seddone (2015a) y De Luca y Fasano (2018). 
Gráfico 6. Participación en las primas del PD (200-2009)

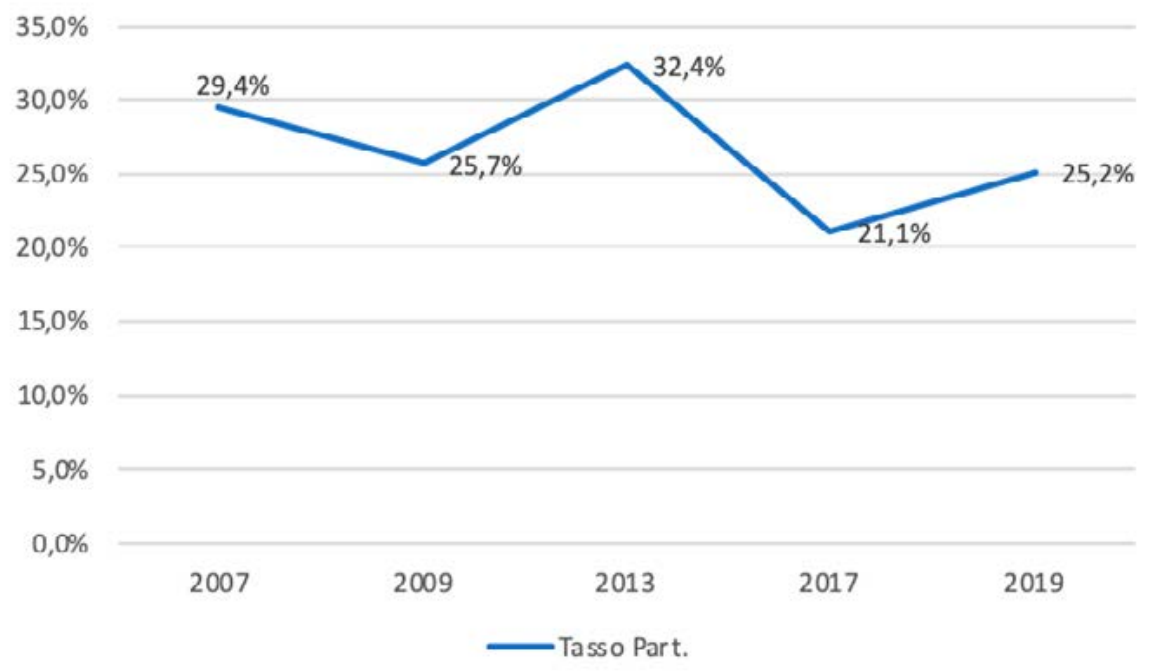

Fuente: elaboración propia.

La mayoría del selectorado del PD la conforman, claramente, votantes de centro-izquierda, si bien merece observar su evolución, entre 2007 y 2017, hacía posiciones de centro, como demuestra la disminución, del $40 \%$ al $34 \%$, de las electores que se identifican con la izquierda. Siempre entre 2007 y 2017, el grupo de los que se declaraban de centro aumentó del 10\% al 15\% (Natale y Fasano, 2017). Sin embargo, en las últimas primarias, celebradas en 2019, la tendencia que había marcado la evolución del selectorado del PD hacia el centro durante una década registra una neta inversión: aquellos que se declaraban de centro disminuían hasta el $13 \%$, mientras que los identificados con la izquierda aumentaban hasta el $42 \%$. Esta recuperación del selectorado de la izquierda, en coincidencia con el cierre de la era renziana, debe atribuirse, sobre todo, a los electores del nuevo secretario, Zingaretti, entre los cuales esta componente resultaba significativamente mayoritaria (De Luca, 2019). Desde 2013, el PD registró una adhesión de participantes declarados de centro-derecha a las primarias de un $3 \%$, y que en gran medida prestó su apoyo a Renzi en las primarias del 2013 y 2017. El peso de este grupo descendió al $1 \%$ en 2019. En definitiva, el partido parece preparado para movilizar, sobre todo, un electorado de centro-izquierda, con mayor atractivo para el electorado centrista en la etapa de Renzi, mientras que las últimas primarias señalan el retorno a un electorado tradicionalmente identificado con la izquierda. 
Al tratarse el PD de un partido centrado en el mecanismo de las primarias para la elección del líder, hasta ayer mismo candidato in pectore del partido a guiar la coalición de centro-izquierda, el rol de los afiliados podría parecer residual en la organización. Sin embargo, gracias al llamado «laudo Migliavacca», que toma el nombre del responsable de organización de DS en tiempos de la secretaría de Piero Fassino, se encontró un compromiso entre selectores y afiliados en el momento de la constitución del PD, que convierte a los últimos un activo importante del partido (Natale y Fasano, 2017). El «laudo Migliavacca", después implementado en el Estatuto del PD, prevé que a los afiliados, a través del voto de los círculos, les sea reconocido el derecho a seleccionar, entre los distintos candidatos a la secretaría, a los participantes finales en las primarias, mientras que a los selectores, junto a los mismos afiliados, se les atribuía el derecho de elegir, mediante su voto en las primarias, al futuro secretario. De este modo, el partido diseñó un procedimiento para la elección del secretario basado en tres fases (Venturino, 2014 y 2018). Los afiliados del PD son los titulares de la oferta política, en modo tal que también son los protagonistas de una suerte de discusión congresual sobre los candidatos y sus plataformas programáticas a través la convención de los círculos (primera fase). Los electores, junto a los afiliados, son, en cambio, los titulares de la demanda política, que se expresa en el voto a los candidatos seleccionados (segunda fase). En base a las mismas reglas, se establecía que en las primarias solo pudiesen participar los primeros tres candidatos más votados en la convención de los círculos. Finalmente, en el caso de que ninguno de los tres candidatos que concurren a las primarias conquistase la mayoría absoluta de los votos, la elección del secretario recaería en la Asamblea Nacional del partido (tercera fase). Cabe subrayar, en todo caso, que en el conjunto de elecciones primarias guiadas por este mecanismo hasta la fecha, la tercera fase no ha sido activada nunca. No obstante, la previsión de esta ulterior fase permitiría corregir casos eventuales de indeterminación en el veredicto de los electores, restituyendo así el cetro a los afiliados.

La evolución de la afiliación al PD, como puede observarse en el gráfico 7, tras una cifra de casi 800000 afiliados en los primeros dos ańos de vida del partido (2008-09), ha ido disminuyendo progresivamente, hasta llegar a su mínimo, con poco más de 370000 inscritos en el bienio 2018-19, una década después de la primera campaña de afiliación. Esta lógica evolutiva solo ha invertido su tendencia en dos ocasiones, en 2013 y 2017, coincidiendo con las primarias que registraron la elección y la reelección de Renzi como secretario del PD, en las que se alcanzaron cerca de 540000 afiliados (Natale y Fasano, 2017). 
Gráfico 7. Evolución de los afiliados al PD (2008-2019)

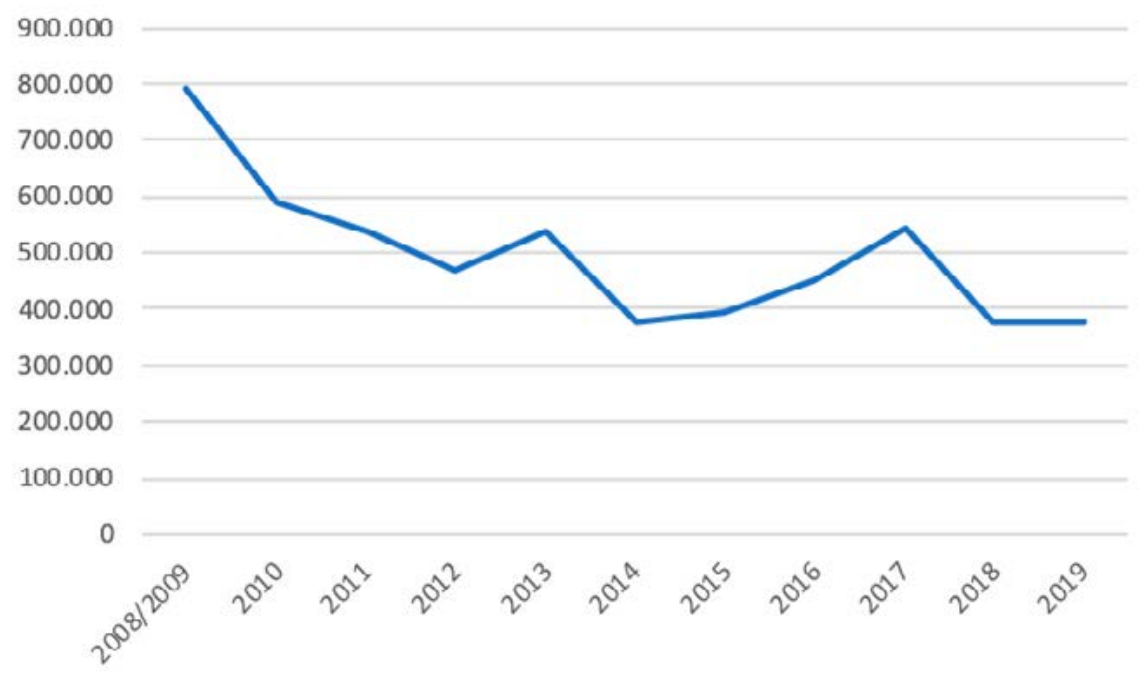

Fuente: elaboración propia.

Cabe destacar, como dato más importante sobre el mecanismo de las primarias del PD, que los afiliados tienen un juicio extremadamente positivo sobre las mismas ${ }^{33}$. Las consideran un aspecto constitutivo de la identidad del partido, atribuyéndole un valor fundamental en su propia experiencia de militancia. Para el $73 \%$ de los afiliados, las primarias contribuyen a mejorar su valoración del partido: tres cuartos de los mismos las consideran un instrumento útil para la renovación de la clase política. Además, algo más del $80 \%$ sostiene que el mecanismo de las primarias no reduce ni el poder ni el rol efectivo de los afiliados en la vida democrática interna del partido. Una valoración que contrasta, de manera sobresaliente, con la preocupación mostrada por una parte del grupo dirigente, el cual, en más de una ocasión, ha sostenido que el recurso al modelo de primarias abiertas a la participación de los electores equivale a desaforar, sustancialmente, la base del partido. En la misma

33 Este dato, como los que siguen sobre las actitudes y valoraciones de los afiliados del PD, han sido recogidos en el marco de un estudio demoscópico realizado por Candidate and Leader Selection, standing group de la Sociedad Italiana de Ciencia Política. El estudio fue realizado entre marzo y abril de 2013, después de las elecciones de 2013, con una muestra de 13650 personas (Sandri y Seddone, 2015b; Fasano y Seddone, 2016). 
línea, el $62 \%$ de los afiliados excluye que las primarias sean fuente potencial de conflicto en el partido y en la coalición del centro-izquierda, mientras que el $66 \%$ sostiene que el método debería ser extendido y utilizado de manera sistemática para la elección de los candidatos a alcaldes y presidentes de región, así como para identificar a los candidatos al Parlamento (Natale y Fasano, $2017)^{34}$. Finalmente, desde el punto de vista de los estilos de militancia cabe reseñar que los exmiembros de DS y La Margherita muestran una tendencia a la movilización política más intensa y continuada, sobre todo en la fase de las primarias, expresada desde la adhesión al comité electoral de alguno de los candidatos, hasta la participación activa en la campaña del candidato escogido. Los nativos del PD, sin embargo, se movilizan en menor medida, limitándose, muchas veces, a la participación en iniciativas puntuales de apoyo al proprio candidato y a la participación en las primarias. No obstante, estos últimos muestran una mayor inclinación a desarrollar otro tipo de actividades de carácter asociativo, con carácter social y comunitario, así como en agrupaciones de carácter recreativo.

\section{CONCLUSIONES: EL PD COMO PARTIDO FALLIDO}

Una docena de años después del nacimiento del PD, las expectativas generadas por su constitución permanecen, en gran medida, insatisfechas, ya que el balance general que se puede hacer de su singladura política no es, ciertamente, positivo. Cambios continuados en el liderazgo, continuas escisiones, divisiones internas permanentes, con su consiguiente reflejo en las variaciones constantes registradas en la línea política, son las características más evidentes que definen su tormentosa trayectoria. El PD ha demostrado ser, hasta ahora, un partido fallido. La prueba más evidente de esta condición la ofrece la evolución de su consenso electoral, el cual evidencia que —más allá de episodios de éxito puntual - la propuesta política encarnada por el PD no ha resultado convincente a los ojos de los electores (Natale y Fasano, 2017).

Como se ha señalado, las escisiones y la inestabilidad interna son dos de las características más acusadas del PD. De la separación original

34 Recordamos que a finales de 2012 el PD organizó una consulta entre sus afiliados y electores para la selección de los candidatos a la Cámara de Diputados y el Senado. Una consulta de condición no vinculante, toda vez que la configuración de los cabezas de lista era decidida por la secretaría del partido y la Dirección Nacional a través del método usual de selección negociada entre las diversas facciones organizadas del partido. 
protagonizada por Gavino Angius y Fabio Mussi, —quienes decidieron, todavía en DS, no formar parte del PD para dar vida a Sinistra Democratica-, hasta la reciente escisión de Renzi, junto a 45 parlamentarios para constituir Italia Viva, la trayectoria del partido registra al menos seis procesos de escisión ${ }^{35}$. La concatenación de estos episodios de ruptura con el partido demuestra, sin que quepa desmentirlo, que las barreras que impiden la salida del PD no son lo suficientemente sólidas. Al menos no lo suficiente como para hacer coincidir el horizonte de supervivencia del partido con los objetivos que el mismo partido se dio en la fase de construcción; a saber: favorecer tanto el desarrollo de un fuerte interés en el mantenimiento del partido como de lealtades organizativas difusas (Panebianco, 1982).

Del mismo modo, la continuada sucesión de secretarios — siete en doce años - ha alimentado la imagen de un partido víctima de una extraordinaria inclinación a destruir a sus mejores hijos, encarnando una suerte de "síndrome de Cronos» (Natale y Fasano, 2017). Veltroni, Franceschini, Bersani, Epifani, Renzi, Martina, Zingaretti: secretarios elegidos directamente por los electores y afiliados, que se han alternado con regentes en las fases de transición, cada uno de los cuales ha desarrollado un estilo de liderazgo propio, ha desplegado su propia estrategia, muchas veces en abierta contradicción con la de su predecesor. Este cuadro indica una dificultad patológica del partido para dotarse de una identidad estable, así como de un grupo dirigente incapaz de sostener unas convicciones y contenidos compartidos en el tiempo (Natale y Fasano, 2017), lo que ha producido, en definitiva, una evolución del partido turbulenta. En el frente externo ha mantenido una relación discontinua con sus electores, mientras que en el frente interno tampoco ha sido capaz de consolidar un horizonte claro y estable de supervivencia para el partido a mediolargo plazo.

En este sentido, el problema del partido fallido se refiere, sobre todo, a un proceso de institucionalización frágil y todavía incompleto ${ }^{36}$. En el acto de

35 Recapitulando las escisiones que han llevado a la formación de nuevas formaciones políticas: Angius y Mussi (Sinistra Democratica); Rutelli y Tabacci (API); Civati (Possibile); Fassina y D’Attore (Sinistra Italiana); D’Alema, Bersani y Speranza (Articolo 1-Movimento Democratico e Progressista).

36 En particular, nos referimos a las características que distinguen el proceso de institucionalización de un partido político según Panebianco (1982), proceso en el que los líderes políticos desarrollan una función crucial, gracias a su contribución decisiva en la definición de las metas ideológico-culturales y la identificación de las referencias sociales de la naciente organización. Además, siempre según Panebianco, que para enfocar esta dimensión se remite a los estudios sobre organizaciones de Selznick (1957), un nuevo partido consigue consolidarse — al punto de completar el 
nacimiento de toda formación política, fase en la que dicho partido se encuentra todavía inmerso en el proceso de construcción, los líderes más prominentes y representativos de la formación desarrollan un rol fundamental. De hecho, son ellos quienes garantizan la elaboración y definición de los objetivos que deben guiar la acción política del nuevo sujeto, quienes definen la cultura política y quienes, diseñando una propuesta programática, contribuyen de manera decisiva a la identificación de su electorado potencial. Este es el proceso por el cual, en el curso del tiempo, un nuevo partido incorpora el complejo de los valores y objetivos derivados de los líderes fundadores, convirtiéndose, progresivamente, en un sujeto dotado de vida propia (Panebianco, 1982). Este proceso, que toma el nombre de institucionalización, hace que la supervivencia del partido se convierta en el principal objetivo de la organización ${ }^{37}$. Los resultados de tal proceso - que se realiza, también, a través de la formación de incentivos selectivos dirigidos a dar continuidad a la organización (Olson, 1983) —, se miden, en definitiva, a través de dos dimensiones: el grado de autonomía del partido respecto al ambiente externo y el grado de interrelación entre los distintos niveles organizativos del partido, gracias a los cuales los órganos centrales (party in central office) consiguen gobernar los procesos decisionales internos, coordinándose con el partido en su implantación territorial (party on the ground) ${ }^{38}$.

Precisamente, en lo tocante al grado de interrelación del partido, la condición de merger party ha constituido un obstáculo para la integración en el PD de los grupos dirigentes con origen en los dos partidos fundadores, DS y La Margherita. A ello se debe unir que no siempre ha existido una plena sintonía entre los órganos centrales (party in central office) y los órganos territoriales (party on the ground). La ausencia de un clara identidad política y el conflicto irresuelto sobre los objetivos y estrategias de partido, constantemente dividido entre la componente postdemocristiana y postcomunista, así como entre las almas radical, socialdemócrata y reformista liberal-democrática, han impedido al partido conseguir un grado suficiente y efectivo de cohesión interna. A su vez, el grado de conflictividad interna, que se extiende del

propio proceso de institucionalización - en el momento en el cual su horizonte de supervivencia tiende, justamente, a coincidir con los objetivos que se ha dado en la fase de construcción, lo que conlleva al desarrollo de lealtades organizativas difusas e intereses comunes en su mantenimiento estable en el curso del tiempo. En referencia particular al proceso de institucionalización del PD, véanse Fasano (2010), Fasano y Martocchia Diodati (2014b), además de Natale y Fasano (2017).

37 Pensemos, a tal propósito, en el proceso que Selznick (1957) ha definido como «heterogenesi de los fines».

38 Cfr. Panebianco (1982). 
centro a la periferia, alimentándose de la lógica competitiva desencadenada por las primarias, ha contrarrestado la puesta en común de las prioridades de la acción política esbozadas por cada liderazgo. En definitiva, nacido el PD, nunca vio la luz, empero, su cultura política.

\section{BIBLIOGRAFIA}

Addario, N. y Fasano, L.M. (2015). La vera innovazione è politica, Il Mulino, 64 (1), 63-68. Almagisti, M., Lanzalaco, L. y Verzichelli, L. (cur.) (2014). La transizione politica italiana. Da Tangentopoli a oggi. Roma: Carocci.

Ariemma, I. (2014). Il tramonto di una generazione. Dalla fine del PCI al Partito Democratico di Renzi. Firenze: Castelvecchi.

Bellucci, P., Maraffi, M. y Segatti, P. (2000). PCI, PDS, DS. La trasformazione dell'identità politica della sinistra di governo. Milano: Donzelli.

Biorcio, R. y Natale, P. (2013). Politica a Cinque stelle. Idee, storia e strategie del movimento di Grillo. Milano: Feltrinelli.

Bordandini, P. y Cartocci, R. (2011). La cultura politica dei delegati nazionali di partito. Polis, 25 (2), 171-204.

Bordandini, P., Di Virgilio, A. y Mulè, R. (2011). I leader degli anni a venire. Atteggiamenti e opinioni dei delegati di partito in Italia. Polis, 25 (2), 159-170.

Calise, M. (2006). Presidentialization, Italian Style. En T. Poguntke e P. Webb (eds.), The Presidentialization of Politics. A Comparative Study of Modern Democracies (pp. 88-106). Oxford: Oxford University Press. Disponible en: https://doi.org/10.1093/0199252017.003.0004.

Cavataio, M. y Fasano, L. M. (2014). Leali, incerti o defezionisti? Atteggiamenti di voto post primarie nei selettori fra il 2007 e il 2013. Quaderni di Scienza politica, 21 (3), 329-349.

Chiapponi, F. (2017). Democrazia, populismo, leadership: il MoVimento 5 Stelle. Novi Ligure: Epoké.

Corbetta, P. y Gualmini, E. (2013). Il partito di Grillo. Bologna: Il Mulino.

De Luca, R. (2019). Il recupero a sinistra di Zingaretti. La collocazione politica dei selettori. Questioni Primarie, 5 (8), 16-17.

- y Fasano, L. M. (2018). Il Partito democratico dei native. Novi Ligure: Epoké.

Drucker, H. M. (1979). Doctrine and Ethos in the Labour Party. London: George Allen and Unwin.

Emanuele, V. y Marino, B. (2019). La partecipazione su base territoriale, Questioni Primarie, $5(8), 6-8$.

Fasano, L. M. (2009). L'Assemblea costituente nazionale del PD. En G. Pasquino (cur). Il Partito democratico. Elezione del segretario, organizzazione e potere (pp. 141-186). Bologna: Bononia University Press.

- (2010). L'Assemblea nazionale del PD. En G. Pasquino y F. Venturino (cur). Il Partito democratico di Bersani. Persone, profilo e prospettive (pp. 35-66). Bologna: Bononia University Press. 
— y Martocchia Diodati, N. (2014a). Il Pd di Renzi, l'ultima occasione. Il Mulino, 63 (1), 45-52.

- y Martocchia Diodati, N. (2014b). L'Assemblea nazionale del Partito democratico. En G. Pasquino y F. Venturino (cur.). Il Partito democratico secondo Matteo (pp. 65-100). Bologna: Bononia University Press.

— , Martocchia Diodati, N. y Natale, P. (2018). L'Assemblea nazionale del Partito democratico. En R. De Luca e L.M. Fasano (cur.). Il Partito democratico dei nativi (pp. 177-196). Novi Ligure: Epoké.

- y Natale, P. (2019). Dal Partito «amalgama» di Veltroni al partito «omnibus» di Zingaretti (2007-2019): ruolo della leadership, forma partito e costruzione della linea politica negli orientamenti dei delegati dell'Assemblea nazionale. En II Convegno annuale della Società italiana di Scienza politica (Lecce, 12-14 settembre), Sezione 9 Elezioni e Comportamento di voto, (Panel 9.2 Leadership and Candidate Selection in Comparative Perspective.

— y Pasini, N. (2014). Tra frammentazione e polarizzazione del sistema politico italiano: interpretazioni e casi empirici. En M. Almagisti, L. Lanzalaco y L. Verzichelli (cur.). La transizione politica italiana. Da Tangentopoli a oggi (pp. 109-142). Roma: Carocci.

- y Seddone, A. (2016). Selecting the Leader, Italian Style. Contemporary Italian Politics, 8 (1), 83-102. Disponible en: https://doi.org/10.1080/23248823.2016.1156313.

Fassino, P. (2018) Pd davvero. Milano: La nave di Teseo.

Floridia, A. (2019). Un partito sbagliato: Democrazia e organizzazione nel Partito Democratico. Firenzce: Castelvecchi.

Funiciello, A. (2012). A vita. Come e perché nel Partito Democratico i figli non riescono a uccidere i padri. Milano: Donzelli.

Gelli, B., Mannarini, T. y Talò, C. (2013). Perdere vincendo. Dal successo delle primarie 2012 all'impasse post-elettorale. Milano: Angeli.

Ignazi, P. y Bordandini, P. (2018). I muscoli di partito. Il ruolo dei quadri intermedi nella politica atrofizzata. Bologna: Il Mulino.

Macaluso, E. (2007). Al capolinea. Controstoria del Partito democratico. Milano: Feltrinelli.

Musella, F. (2015). Personal leaders and party change: Italy in comparative perspective, Italian Political Science Review. Rivista Italiana di Scienza Politica, 45 (3), 227-247. Disponible en: https://doi.org/10.1017/ipo.2015.19.

- y Webb, P. (2015). The Revolution of Personal Leaders. Italian Political Science Review. Rivista Italiana di Scienza Politica, 45 (3), 223-226. Disponible en: https://doi. org/10.1017/ipo.2015.20.

Natale, P. (2019). L'Assemblea nazionale del Partito democratico. En S. Rombi e F. Serricchio (cur.). L'elezione di Zingaretti. La rivincita del partito? (pp. 229-241). Novi Ligure: Epoké.

- y Fasano, L. M. (2017). L'ultimo partito. Dieci anni di Partito democratico. Torino: Giappichelli.

Olson, M. (1983). La logica dell'azione collettiva. Milano: Feltrinelli.

Panebianco, A. (1982) Modelli di partito. Organizzazione e potere nei partiti politici. Bologna: Il Mulino.

Pasquino, G. (cur.) (2009). Il Partito democratico. Elezione del segretario, organizzazione e potere. Bologna: Bononia University Press. 
— y Venturino, F. (cur.) (2010). Il Partito democratico di Bersani. Persone, profilo e prospettive. Bologna: Bononia University Press.

- y Venturino, F. (cur.) (2014). Il Partito democratico secondo Matteo. Bologna: Bononia University Press.

Passarelli, G. (2015). The Presidentialization of Political Parties. Organizations, Institutions and Leaders. NewYork:Macmillan. Disponibleen:https://doi.org/10.1057/9781137482464.

— y Tuorto, D. (2018). La Lega di Salvini. Estrema destra di governo. Bologna: Il Mulino.

Poguntke, T. y Webb, P. (cur.) (2006). The Presidentialization of Politics. A Comparative Study of Modern Democracies. Oxford: Oxford University Press.

Raniolo, F. (2011). Partecipazione e partiti: la prospettiva dei delegati congressuali. Polis, 25 (2), 235-262.

Reif, K., Cayrol, R. y Niedermayer, O. (1980). National Political Parties' Middle-Level Elites and European Integration. European Journal of Political Research, 8 (1), 91-112. Disponible en: https://doi.org/10.1111/j.1475-6765.1980.tb00740.x.

Salvati, M. (2003). Il Partito democratico. Alle origini di unidea politica. Bologna: Il Mulino.

Sandri, G. y Seddone, A. (cur.) (2015a). The Primary Game. Primary Elections and the Italian Democratic Party. Novi Ligure: Epoké.

- (2015b). In Members' Shoes: Members' Opinions on Intra-Party Democracy and Primary Elections. En G. Sandri y A. Seddone (cur.). The Primary Game. Primary Elections and the Italian Democratic Party (pp. 129-152). Novi Ligure: Epoké.

Schwartz, S. H. (1992). Universals in the Content and Structures of Values. Theory and Empirical Tests in 20 Countries. Advances in Experimental Social Psychology, 25, 1-65. Disponible en: https://doi.org/10.1016/S0065-2601(08)60281-6.

- (1994). Are there universal aspects in the structure and contents of human values. Journal of Social Issues, 50 (4), 19-45. Disponible en: https://doi.org/10.1111/j.15404560.1994.tb01196.x.

Selznick, P. (1957). La leadership nelle organizzazioni. Milano: Angeli.

Tedeschi, S. (2018). Il Partito democratico. Origine, organizzazione e identità. Novi Ligure: Epoké.

Venturino, F. (2014). Il Partito democratico e la selezione del leader: le regole della competizione. En G. Pasquino y F. Venturino (cur.). Il Partito democratico secondo Matteo (pp. 13-28). Bologna: Bononia University Press.

- (2018) La selezione del leader: le regole della competizione e i candidati. En R. De Luca y L. M. Fasano (cur.). Il Partito democratico dei native (pp. 17-30). Novi Ligure: Epoké. 